\title{
Structures for Epistemic Logic
}

Nick Bezhanishvili and Wiebe van der Hoek

\section{Introduction}

Epistemic modal logic in a narrow sense studies and formalises reasoning about knowledge. In a wider sense, it gives a formal account of the informational attitude that agents may have, and covers notions like knowledge, belief, uncertainty, and hence incomplete or partial information. As is so often the case in modal logic, such formalised notions become really interesting when studied in a broader context. When doing so, epistemic logic in a wider sense in fact relates to most of the other chapters in this book. What if we add a notion of time or action (Chapter 21): how does an agent revise its beliefs (cf. Chapter 8), or update its knowledge (Chapter 7)? And even if we fix one of the notions of interest, say knowledge, if there are many agents, how can we ascribe some level of knowledge to the group, and how do we represent knowledge of one agent about the knowledge (or ignorance, for that matter) of another (cf. Section 2.1)? What are reasonable requirements on the interaction between knowledge and strategic action (Chapter 15), and how is uncertainty dealt with in more general, qualitative models of agency (Chapter 12)?

Hintikka, notably through [66], is broadly acknowledged as the father of modern epistemic modal logic. Indeed, [66] gives an account of knowledge and belief based on Kripke models. In a nutshell, crucial for this semantics is the notion of a set of states or worlds, together with a binary relation for each agent, determining which worlds 'look the same', for the agent, or 'carry the same information'. Many disciplines realised the importance of the formalisation of knowledge, using Kripke semantics (or a close relative of it). Examples of such disciplines are Artificial Intelligence (notably Moore's [89] on actions and knowledge) philosophy (Hintikka's [67]), game theory (see Aumann's formalisation of common knowledge, [4]. Au-

Nick Bezhanishvili

Department of Philosophy, Utrecht University, e-mail: N.Bezhanishvili@uu.nl

Wiebe van der Hoek

Department of Computer Science, University of Liverpool, e-mail: wiebe@ liv . ac . uk 
mann's survey [5] on interactive epistemology can easily be recast using a Kripke semantics), and agents (the underlying semantics of the famous BDI approach by Rao and Georgeff for instance ([93]) is based on Kripke models). For more references to those disciplines, we refer to the chapters on the relevant topics in this book.

Another important aspect of this chapter is to review the neighbourhood and topological semantics of epistemic and doxastic logic. Topological semantics of modal logics originates from the ground-laying work of MacKinsey and Tarski [85]. In recent years there has been a surge of interest in this semantics not least because of its connection to epistemic and doxastic logic. Van Benthem (not surprisingly) has been in the centre of the recent developments in the area.

In short, the aim of this chapter is to explain some of the most popular semantic structures used to model informational attitudes, and at several places we have plenty of opportunity to point at van Benthem's contribution to the field. In fact, Johan's work spins over the different semantics of epistemic logic that we discuss here. It builds bridges between many different areas. Therefore, we cannot think of a better place for publishing this chapter than a volume dedicated to Johan's contributions.

The chapter is organised as follows. In Section 2, we briefly introduce a family of modal epistemic languages that are interpreted on the structures to be discussed. We also discuss the most popular axiom systems for multi-agent knowledge and belief. Then, in Section 3, we introduce probably the most celebrated structures for epistemic logic, i.e., epistemic Kripke structures. Based on Kripke models, we then add a temporal or a computational component, and also provide a 'grounded' semantics for knowledge. In Section 4 we consider 'non-standard', or 'generalised' semantics for knowledge and belief, i.e., semantics that are not based on an underlying relation on the sets of states. In particular, we discuss here neighbourhood semantics and topological semantics. In Section 5, we conclude.

\section{Epistemic Logic: Language and Axiom Systems}

Let us first agree on a formal language for reasoning about information of agents.

\section{Definition 1 (A Suite of Modal Epistemic Languages).}

We assume a set At $=\left\{p, q, p_{1}, \ldots\right\}$ of atomic propositions, a set of agents $\mathrm{Ag}=\{1, \ldots, m\}$ and a set of modal operators Op. Then we define the language $\mathrm{L}(\mathrm{At}, \mathrm{Op}, \mathrm{Ag})$ by the following BNF:

$$
\varphi:=p|\neg \varphi|(\varphi \wedge \varphi) \mid \square \varphi
$$

where $p \in$ At and $\square \in$ Op.

Abbreviations for the connective $\vee$ ('disjunction'), $\rightarrow$ ('implication') and $\leftrightarrow$ ('equivalence') are standard. Moreover the dual $\diamond \varphi$ of an operator $\square \varphi$ is defined 
as $\neg \square \neg \varphi$. Typically, the set Op depends on Ag. For instance, the language for multi-agent epistemic logic is $\mathrm{L}(\mathrm{At}, \mathrm{Op}, \mathrm{Ag})$ with $\mathrm{Op}=\left\{K_{a} \mid a \in \mathrm{Ag}\right\}$, that is, we have a knowledge operator for every agent. $K_{a} \varphi$ reads 'agent $a$ knows that $\varphi$ ', so that $K_{a} \varphi \vee K_{a} \neg \varphi$ would indicate that agent $a$ knows whether $\varphi$ (which should be contrasted with the 'propositional' validity $\left(K_{a} \varphi \vee \neg K_{a} \varphi\right)$ and the 'modal' validity $K_{a}(\varphi \vee \neg \varphi)$ ). The dual of $K_{a}$ is often written $M_{a}$. So for instance $M_{a} \varphi \wedge M_{a} \psi \wedge$ $\neg M_{a}(\varphi \wedge \psi)$ says that agent $a$ holds both $\varphi$ and $\psi$ to be possible, although he knows that $\varphi$ and $\psi$ hold not both. For a language in which one wants to study interaction properties between knowledge and belief, we would have $\mathrm{Op}=\left\{K_{a}, B_{a} \mid a \in \mathrm{Ag}\right\}$. A typical interaction property in such a language would be

$$
K_{a} \varphi \rightarrow B_{a} \varphi
$$

but of course not the other way around, since one would like the two notions of knowledge and belief not to collapse: [74] for instance assume (1) and $B_{a} \varphi \rightarrow$ $K_{a} B_{a} \varphi$ as an axiom, but warn that 'the interesting formula $B_{a} \varphi \rightarrow B_{a} K_{a} \varphi$ is not included in our system', the reason for it being that knowledge and belief would become the same. This lead [70] to study 'how many' interaction between the two notions one can allow before they become the same: the latter study is in fact an application of correspondence theory, a notion developed by van Benthem in his $\mathrm{PhD}$ thesis [8], to which we will come back later (note also that Chapter 23 in this volume is dedicated to this topic).

So what then are the properties of knowledge and belief proper, and how do the two notions differ? To start with the latter question, in modal logic it is often assumed that knowledge is veridical, where belief is not. In other words, knowledge satisfies $K_{a} \varphi \rightarrow \varphi$ as a principle, while for belief, it is consistent to say that $a$ believes certain $\varphi$, although $\varphi$ is in fact false. Of course, agent $a$ will not consider this a possibility: indeed, in the 'standard' logic for belief, we have that $B_{a}\left(B_{a} \varphi \rightarrow \varphi\right)$ is valid. The axioms Taut and $\mathbf{K}_{\square}$ and the inference rules MP and $\mathbf{N e c}_{\square}$ form the

\begin{tabular}{|l|l|}
\hline \multicolumn{1}{|c|}{ Basic modal properties } & Epistemic and Doxastic properties \\
\hline Taut all instantiations of propositional tautologies & $\mathbf{D} \neg \square \varphi$ \\
$\mathbf{K}_{\square} \quad \square(\varphi \rightarrow \psi) \rightarrow(\square \varphi \rightarrow \square \psi)$ & $\mathbf{T} \quad \square \varphi \rightarrow \varphi$ \\
\hline $\mathbf{M P} \quad$ From $\varphi$ and $\varphi \rightarrow \psi$, infer $\psi$ & $\mathbf{4} \quad \square \varphi \rightarrow \square \square \varphi$ \\
$\mathbf{N e c}_{\square}$ From $\varphi$, infer $\square \varphi$ & $\mathbf{5} \quad \neg \square \varphi \rightarrow \square \neg \square \varphi$ \\
\hline
\end{tabular}

Table 1 Basic modal and epistemic and doxastic axioms.

modal logic $K$. For knowledge, one then often adds veridicality (T), and positive(4) and negative introspection (5). For belief, veridicality is usually replaced by the weaker axiom consistency (D). If there are $m$ agents (i.e., $m$ knowledge operators $\left.K_{1}, \ldots, K_{m}\right)$, the axioms of $K+\{\mathbf{T}, \mathbf{4}\}$ are referred to as $S 4_{m}$, the axioms of $K+$ $\{\mathbf{T}, \mathbf{4}, \mathbf{5}\}$ are referred to as $S 5_{m}$, and we call the agents in the latter case epistemic agents. The arguably most popular logic for belief $K+\{\mathbf{D}, \mathbf{4 , 5}\}$ is usually denoted 
$K D 45_{m}$. In fact, agents that are veridical and negatively introspective must already be positively introspective (and hence epistemic agents), i.e., $K+\{\mathbf{T}, \mathbf{5}\} \vdash \mathbf{4}$.

A normal modal logic is a set of formulas $L$ containing all instances of axioms of $K$ and closed under the rules MP and $\mathbf{N e c}_{\square}$. We write $L \vdash \varphi$ if $\varphi$ is a theorem of $L$.

\subsection{Multi-agent Notions}

To speak with van Benthem, One is a lonely number ([10]), and the notions of knowledge and belief become only more interesting in a multi-agent setting (and, as [10] also argues, in a dynamic setting, but for this, we refer to Chapter 7). Let $A \subseteq \mathrm{Ag}$ be a set of agents. One can then introduce an operator that says that everybody in $A$ knows something: $E_{A} \varphi=\bigwedge_{a \in A} K_{a} \varphi$ (instead of $E_{\mathrm{Ag}}$, write $E$ ). Obviously, this does not expand the logic's expressivity, but it does indeed decrease the descriptive complexity ([49]): even in $S 5_{m}$, having the operator $E_{A}$ (if $|A| \geq 4$ ) makes the language more succinct.

One could in a similar way, using disjunctions, define a notion of 'somebody knows'. However, arguably a more interesting (and logically stronger) notion is that of distributed knowledge $D_{A} \varphi$ in a group $A$ of $\varphi$. For instance, if $a$ knows that every modal logician is interested in epistemic logic, and $b$ knows that van Benthem is a modal logician, then there is distributed knowledge among $a$ and $b$ that van Benthem is interested in epistemic logic, even if none of the agents needs to know this.

\begin{tabular}{|c|c|}
\hline Everybody's and Common Knowledge & Distributed Knowledge \\
\hline$\overline{\mathbf{E}} \quad E \varphi \leftrightarrow \bigwedge_{a \in \mathrm{Ag}} K_{a} \varphi$ & $\bigvee_{a \in \mathrm{Ag}} K_{a} \varphi \rightarrow D \varphi$ \\
\hline $\mathbf{K}_{\mathbf{C}} \quad C(\varphi \rightarrow \psi) \rightarrow(C \varphi \rightarrow C \psi)$ & $D(\varphi \rightarrow \psi) \rightarrow(D \varphi \rightarrow D \psi)$ \\
\hline $\operatorname{Mix} \quad C \varphi \rightarrow(\varphi \wedge E C \varphi)$ & $D \varphi \rightarrow \varphi$ \\
\hline Ind $\quad C(\varphi \rightarrow E \varphi) \rightarrow(\varphi \rightarrow C \varphi)$ & $\neg D \varphi \rightarrow D \neg D \varphi$ \\
\hline $\mathbf{N e c}_{\mathbf{C}}$ From $\varphi$, infer $C \varphi$ & $\mathbf{N e c}_{\mathbf{D}}$ From $\varphi$, infer $D \varphi$ \\
\hline
\end{tabular}

Table 2 Axioms and inference rules for group-, common- and distributed knowledge.

Arguably the most interesting epistemic group notion is that of common knowledge of a group. Common knowledge of $\varphi$ is supposed to mean that everybody knows $\varphi$, and moreover, everybody knows that, and everybody knows .... If our language would allow for infinite formulas, common knowledge would be captured by the infinite conjunction

$$
E \varphi \wedge E E \varphi \wedge E E E \varphi \wedge \ldots
$$

Phrased negatively, $\varphi$ is not common knowledge as long as somebody considers it possible that somebody considers it possible that ... somebody considers it possible that $\varphi$ is false. Common knowledge explains why social laws (like a green traffic 
light) work: when approaching a green light, I not only know that I have preference, but I also know that you know this, and that you know that I know it, etc. In games, common knowledge of rationality explains why certain strategies can be singled out as being in equilibrium (see Chapter 15). The axioms for common knowledge are $\mathbf{K}_{\mathbf{C}}, \mathbf{M i x}$, Ind and inference rule $\mathbf{N e c}_{\mathbf{C}}$ from Table 2. If $L_{m}$ is a logic with $m$ operators $K_{a}$, then adding the axioms $\mathbf{E}, \mathbf{K}_{\mathbf{C}}, \mathbf{M i x}$, Ind and rule $\mathbf{N}_{\mathbf{C}}$ is denoted by $L_{m}^{C}$. Similarly for $L_{m}^{D}$ for $L$ with the axioms for distributed knowledge added. Sometimes, the axiom Ind is replaced by the inference rule

$$
\text { From } \varphi \rightarrow E(\psi \wedge C \varphi) \text { infer } \varphi \rightarrow C \psi
$$

Axioms and inference rules for the epistemic group notions discussed here are given in Table 2. They are usually added to $S 5_{m}$. Notions of common belief and distributed belief also exist: for those, one usually adds slightly weaker axioms.

As for instance explained by van Benthem in [12], we can define common knowledge $C \varphi$ also as a fixed point of the following operator:

$$
\varphi \wedge E x
$$

A fixed point $\psi$ of this operator satisfies $\psi=\varphi \wedge E \psi=\varphi \wedge E(\varphi \wedge E \psi) \ldots$ in which one recognises the Mix axiom. Moreover, the Ind axiom states we have a greatest fixed point, which can be obtained by iterated application of the operator to $T$, giving $\varphi \wedge E \top, \varphi \wedge E(\varphi \wedge E \top), \varphi \wedge E(\varphi \wedge E(\varphi \wedge E \top))$, etc., see Section 3.1 for more details.

Common knowledge is obviously the strongest epistemic notion discussed here, while distributed knowledge is the weakest (see (4)). As a consequence, common knowledge will be typically obtained for 'weak' formulas $\varphi$ only (even if everybody in a group knows that Santa Claus does not exist, this does not have to be common knowledge), while distributed knowledge may pertain to 'strong' statements (no matter how large the group is, there is distributed knowledge about the fact whether there are two members sharing their birthday). In terms of [46], common knowledge is what 'any fool' knows, while distributed knowledge characterises what the 'wise man' knows. It is not difficult to see that when one adds the principles of Table 2 to $S 5_{m}$, both the wise man and the fool are epistemic agents.

$$
C \varphi \Rightarrow E \varphi \Rightarrow K_{a} \varphi \Rightarrow D \varphi \Rightarrow \varphi
$$

\subsection{Knowledge and Time}

One of the most prominent themes in van Benthem's work in the last two decades is that of dynamics. There is a complete chapter (Chapter 7) in this volume dedicated to Dynamic Epistemic Logic. A simple setting to study dynamics of epistemics is obtained by combining temporal and epistemic logic (temporal logic is the subject of Chapter 21). Popular temporal models of agency are linear time models or else 
trees. For both, one can use Linear Time Logic (LTL) to reason about them. In the latter case, properties of the tree are those true on all of its branches (in CTL, one can quantify over branches as well). In LTL, one uses operators for $\bigcirc \varphi$ ('in the next state'), $\square$ ('always in the future'), $\diamond$ ('some time in the future') and $U$ (where $\varphi U \psi$ denotes ' $\varphi$ holds until $\psi$ is true'). When we want to refer to the memory of the agents, also past-time operators are used, allowing for $\boldsymbol{\varphi} \varphi$ ('in the previous moment'), ('always in the past') and ('some time in the past').

Some axioms for linear temporal time logic with future operators are given in Table 3. Let us call the logic consisting of them $L T L$. The future operators 'some time' and 'always' can be defined as $\nabla \varphi=\neg \varphi U \varphi$ and $\square \varphi=\neg \diamond \neg \varphi$, respectively. Axiom $\mathbf{T}_{\mathbf{2}}$ says that $\bigcirc$ is functional (this is the $\leftarrow$-direction, saying there is at most one next state) and serial (the $\rightarrow$-direction, saying there is at least one next state). $\mathbf{T}_{\mathbf{3}}$ defines until: ' $\varphi$ until $\psi$ ' is equivalent to saying that 'either $\neg \psi$, or $\varphi$ holds while in the next state, $\varphi$ until $\psi$ '. The rule RT explains how $\neg(\varphi U \psi)$ can be inferred, and this rule is reminiscent of the induction rule (RInd) for common knowledge (cf. [46, Theorem 8.1.1(e)]).

\begin{tabular}{|ll|l|}
\hline \multicolumn{1}{|c|}{ Next } & \multicolumn{1}{|c|}{ Next and Until } \\
\hline $\mathbf{K}_{\bigcirc} \bigcirc(\varphi \rightarrow \psi) \rightarrow(\bigcirc \varphi \rightarrow \bigcirc \psi)$ & \\
$\mathbf{T}_{\mathbf{2}}$ & $\bigcirc \neg \varphi \leftrightarrow \neg \bigcirc \varphi$ & $\mathbf{T}_{\mathbf{3}} \varphi U \psi \leftrightarrow \psi \vee(\varphi \wedge \bigcirc(\varphi U \psi))$ \\
\hline $\mathbf{N e c}_{\bigcirc}$ & From $\varphi$, infer $\bigcirc \varphi$ & RT From $\varphi^{\prime} \rightarrow \neg \psi \wedge \bigcirc \varphi$, infer $\varphi^{\prime} \rightarrow \neg(\varphi U \psi)$ \\
\hline
\end{tabular}

Table 3 Axioms and inference rules for linear temporal logic with next and until.

Similarly to common knowledge, the until operator also allows a fixed point definition as the least fixed point of $\psi \vee(\varphi \wedge \diamond x)$. As always, things become more interesting when we look at properties that relate the modalities (for knowledge and time in this case) that we have. Typical mix properties for knowledge and time are then for instance

$$
K_{a} \bigcirc \varphi \rightarrow \bigcirc K_{a} \varphi \& \bigcirc K_{a} \varphi \rightarrow K_{a} \bigcirc \varphi \quad \text { (perfect recall (PR) \& no surprise (NS)) }
$$

NS is sometimes called no learning: it expresses that everything that one will know in the next state, is currently already known to hold next. Readers interested in these notions should also consult Chapter 21 by Goranko and Pacuit in this volume.

\section{Relational Epistemic Structures for Knowledge}

We now present a semantics for our formal language, based on Kripke models. 


\subsection{Kripke Models}

Definition 2 (Kripke models and epistemic models). A Kripke model $M$ for $\mathrm{L}(\mathrm{At}, \mathrm{Op}, \mathrm{Ag})$ is a tuple $\langle S, R, V\rangle$ where $S$ is a set of states, or worlds, $R$ associates each $\square \in$ Op with an accessibility relation $R(\square) \subseteq S \times S$. Rather than $(s, t) \in R(\square)$ we write $s R_{\square} t$. Finally, $V$ assigns to each atom $p \in$ At a set of states $V(p) \subseteq S$ : those are the states in $M$ where $p$ is true. A tuple $\langle S, R\rangle$ is called a frame. For $M=\langle S, R, V\rangle$, we will sloppily write $s \in M$ for $s \in S$. Truth of $\varphi$ in a pair $M, s$ (with $s \in M$ ) is then defined as follows:

$$
\begin{array}{lll}
M, s \models p & \text { iff } & s \in V(p) \\
M, s \models \varphi \wedge \psi & \text { iff } & M, s \models \varphi \text { and } M, s \models \psi \\
M, s \models \neg \varphi & \text { iff } & \text { not } M, s \models \varphi \\
M, s=\square \varphi & \text { iff } & \text { for all } t \text { such that } s R_{\square} t, M, t \models \varphi
\end{array}
$$

For $F=\langle S, R\rangle$, the notion $F=\varphi$ is defined as $\forall V, \forall s,\langle S, R, V\rangle, s=\varphi$. In that case, we say that $\varphi$ is valid in $F$. We write $F=L$, if $F=\varphi$ for each $\varphi \in L$. If there exists $s \in S$ and a valuation $V$, such that $\langle S, R, V\rangle, s \models \varphi$, then we say that $\varphi$ is satisfiable in $F$. If $\Gamma$ is a set of formulas, we say $\Gamma$ is satisfiable in $F$ if there is $s \in S$ and a valuation $V$, such that $\langle S, R, V\rangle, s=\varphi$ for each $\varphi \in \Gamma$. Validity of $\varphi$ on a model $M$ is defined as $M, s=\varphi$ for all $s \in M$. The class of all Kripke models $\langle S, R, V\rangle$ with $m$ accessibility relations $R(\square)$ is denoted $\mathcal{K}_{m}$.

Let $\mathcal{C}$ be some class of models. If $M \models \varphi$ for each $M \in \mathcal{C}$, then we say that $\varphi$ is valid in $\mathcal{C}$, and write $\mathcal{C}=\varphi$. Examples of classes of models are $\mathcal{K}_{m}$ (all Kripke models with $m$ relations), $\mathcal{S} 4_{m}$ (models with $m$ relations, all being reflexive and transitive), $\mathcal{K D} 45_{m}$ (all relations being serial, transitive and Euclidean) and $\mathcal{S} 5_{m}$ (all relations are equivalence relations). Also, $\mathcal{U}_{m}$ is the class of models where all $m$ relations are the universal relation. If $\mathcal{C}_{m}$ is class of models for $m$ agents, $\mathcal{C}_{m}^{C}$ is the class obtained by adding a relation $R_{C}$, which is the transitive closure of the union of the $m$ relations. Likewise, $\mathcal{C}_{m}^{D}$ has a relation $R_{D}$ which is the intersection of the relations in the model.

If Op contains one modal operator for each agent, we often write $R_{a}$ rather than for instance $R_{K_{a}}$ or $R_{B_{a}}$. When all the operators are epistemic operators $K_{a}$, we write $\sim_{a}$ for $R_{K_{a}}$, and we assume that $\sim_{a}$ is an equivalence relation. A model with such relations is called an epistemic model, and will be denoted $M=\langle S, \sim, V\rangle$. A pair $M, s$ is also called a pointed Kripke model or pointed epistemic model. So $\mathcal{S} 5_{m}$ represents the class of all epistemic models.

In epistemic models, the interpretation of $s \sim_{a} t$ is that 'states $s$ and $t$ look similar for $a$ ', or 'in $s$ and $t$, agent $a$ has the same information', or, 'given state $s$, agent $a$ considers it possible that the state is $t$ '. These informal readings make it plausible that $\sim_{a}$ is an equivalence relation indeed.

An extremely simple multi-agent scenario involving two agents $a$ and $b$ and one atom $p$ is given in Figure 1. The pointed model $M_{1}, s_{1}$ models a situation where it is given that " $p$, but $a$ and $b$ don't know it". Let us denote this scenario by $\sigma$. Alternative models for the same scenario are given in Figure 2. In our representation 
of such a model, states in which $p$ is true are denoted with a thick circle, and a line between two states labeled with an agent means that the two states are similar for that agent - we omit reflexive arrows which are supposed to be present in all states.

Fig. 1 A simple two-agent

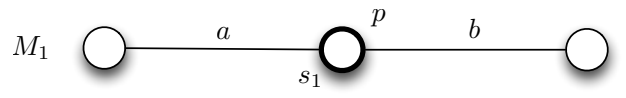
one-atom scenario

We already mentioned van Benthem's pioneering work in Correspondence Theory $[8,24]$. This theory establishes a formal connection between first-order properties of the accessibility relation on the one hand, and axioms or formula schemes, on the other. For instance, the axiom $\mathbf{T}$ corresponds to reflexivity, $\mathbf{4}$ to transitivity and 5 corresponds to the underlying accessibility relation being Euclidean. Since a relation that is reflexive, transitive and Euclidean is an equivalence relation, this then helps us establish that the logic $S 5_{m}$ is sound and complete wrt epistemic models (the doxastic logic $K D 45_{m}$ is sound and complete wrt models where the accessibility relations $R_{B_{a}}$ are serial, transitive and Euclidean). See also Section 3.2, in particular Theorem 2.

By way of illustration of a proof of correspondence, let us follow [11] to show the correspondence between $\mathbf{4}$ and transitivity.

Fact 1 (Fact 1.1 [11]) $F, s \models \square p \rightarrow \square \square p$ iff $F$ 's accessibility relation $R$ is transitive at the point s: i.e., $F, s \models \forall y z((s R y \& y R z) \Rightarrow s R z)$.

Proof. If the relation is transitive, $\square p \rightarrow \square \square p$ clearly holds under every valuation. Conversely, let $F, s=\square p \rightarrow \square \square p$. It means that this axiom holds for every valuation $V$, so in particular when $V(p)=\{y \mid s R y\}$. For this $V$, the antecedent of the model formula holds at $s$, and hence so does $\square \square p$. By definition of $V$, this implies that $R$ is transitive.

Fig. 2 Four 'different' models $M_{i}, s_{i}$ for $p \wedge \neg K_{a} p \wedge \neg K_{b} p$. States where $p$ is true have a thick circle

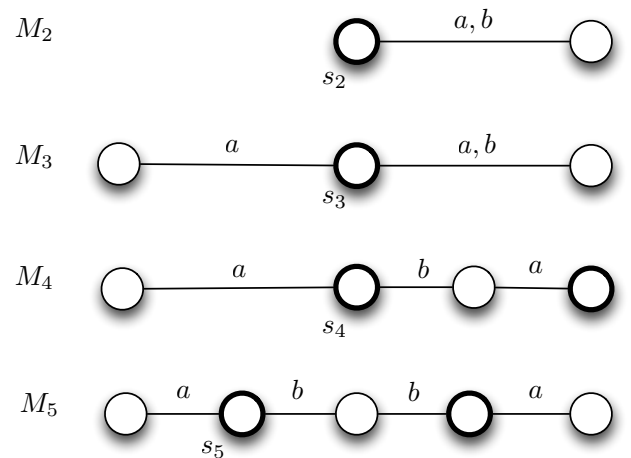


Given an epistemic model $\langle S, \sim, V\rangle$, it turns out that the group epistemic notions $E, C$ and $D$ can all be interpreted as modal operators with respect to some binary relation that is defined in terms of the individual relations $\sim_{a}$. More precisely, the operator $E$ is the necessity operator for the relation $\sim_{E}=\cup_{a \in \mathrm{Ag}} \sim_{a}$ : in order for $E \varphi$ to be true at $M, s$, the formula $\varphi$ needs to be true in all successors of $s$, no matter which agent we choose. ${ }^{1}$ In Figure 1 for instance, we have $M_{1}, s_{1} \models E M_{a} \neg p$ (both $a$ and $b$ know that $a$ considers a $\neg p$-state possible) while $M_{1}, s_{1} \models \neg E M_{a} p$ (since $b$ considers it possible that $a$ knows $\neg p$ ). One can also use correspondence theory to see that $D$ can be interpreted as the modal operator for a relation $\sim_{D}$, with $\sim_{D} \subseteq \cap_{a \in \mathrm{Ag}} \sim_{a}$. At the end of Section 3.2, we will argue that for completeness, one can even replace the ' $\subseteq$ ' by ' $=$ '. In terms of Figure 1 again, we have $M_{1}, s_{1} \models D p$.

For common knowledge, the corresponding property is not first order definable, but van Benthem explains in [11] how it corresponds with a property in First-Order Logic with Least Fixed Points, see also [17].

We briefly recall the semantics of modal $\mu$-calculus (e.g., [34]), skipping some well-known details. The formulas of modal $\mu$-calculus are modal formulas extended with the formulas of type $\mu x \varphi$ and $v x \varphi$ for $\varphi$ positive in $x$ (i.e., if each occurrence of $x$ is under the scope of an even number of negations). Let $\langle S, R\rangle$ be a Kripke frame. For each modal $\mu$-formula $\varphi$ and a valuation $V$, we define the semantics $\llbracket \varphi \rrbracket_{V}$ of $\varphi$ by induction on the complexity of $\varphi$. If $\varphi$ is a propositional variable, a constant, or is of the form $\psi \wedge \chi, \psi \vee \chi, \neg \psi, \square \psi$ or $\diamond \psi$, then the semantics of $\varphi$ is defined as above. For each valuation $V$, we denote by $V_{x}^{U}$ a new valuation such that $V_{x}^{U}(x)=U$ and $V_{x}^{U}(y)=V(y)$ for each propositional variable $y \neq x$ and $U \in \mathcal{P}(S)$.

Let $\varphi$ be positive in $x$, then

$$
\begin{aligned}
& {[\mu \mu x \varphi]_{V}=\bigcap\left\{U \in \mathcal{P}(S): \llbracket[\varphi]_{V_{x}^{U}} \subseteq U\right\} .} \\
& {\left[\operatorname{vx} \varphi \rrbracket_{V}=\bigcup\left\{U \in \mathcal{P}(S): \llbracket[\varphi]_{V_{x}^{U}} \supseteq U\right\} .\right.}
\end{aligned}
$$

We will skip the index $V$ if it is clear from the context. Note that $\llbracket \mu x \varphi]_{V}$ and $\llbracket v \times \varphi]_{V}$ are, respectively, the least and greatest fixed points of the map $f_{\varphi, V}$ : $\mathcal{P}(S) \rightarrow \mathcal{P}(S)$ defined by $\left.f_{\varphi, V}(U)=\llbracket \varphi\right]_{V_{x}^{U}}$. That $\varphi$ is positive in $x$ guarantees that $f_{\varphi, V}$ is monotone. Therefore, by the celebrated Knaster-Tarski theorem these fixed points exist and are computed as in (5) and (6). The least and greatest fixed points can also be reached by iterating the map $f_{\varphi, V}$. In particular, for an ordinal $\alpha$ we let $f_{\varphi, V}^{0}(\emptyset)=\emptyset, f_{\varphi, V}^{\alpha}(\emptyset)=f_{\varphi, V}\left(f_{\varphi, V}^{\beta}(\emptyset)\right)$ if $\alpha=\beta+1$, and $f_{\varphi, V}^{\alpha}(\emptyset)=\bigcup_{\beta<\alpha} f_{\varphi, V}^{\beta}(\emptyset)$, if $\alpha$ is a limit ordinal, and we let $f_{\varphi, V}^{0}(S)=S, f_{\varphi, V}^{\alpha}(S)=f_{\varphi, V}\left(f_{\varphi, V}^{\beta}(S)\right)$ if $\alpha=\beta+1$, and $f_{\varphi, V}^{\alpha}(S)=\bigcap_{\beta<\alpha} f^{\beta}(S)$, if $\alpha$ is a limit ordinal. Then $[\mu x \varphi]_{V}=f_{\varphi, V}^{\alpha}(\emptyset)$, for some ordinal $\alpha$ such that $f_{\varphi, V}^{\alpha+1}(\emptyset)=f_{\varphi, V}^{\alpha}(\emptyset)$ and $[v x \varphi]_{V}=f_{\varphi, V}^{\alpha}(S)$, for some ordinal $\alpha$ such that $f_{\varphi, V}^{\alpha+1}(S)=f_{\varphi, V}^{\alpha}(S)$.

Thus, we have two different ways of computing fixed point operators resulting in the same semantics. As we will see in the next section this is no longer the case in topological semantics. Now we have all the formal machinery for giving a fixed

\footnotetext{
${ }^{1}$ For easy of readability, we give the group notions with $A=\mathrm{Ag}$ : cases for $A \subseteq \mathrm{Ag}$ are similar.
} 
point definition of common knowledge. We let

$$
C \varphi=v x(\varphi \wedge E x) .
$$

A fixed point formula $\mu x \varphi(v x \varphi)$ is called constructive if the least (greatest) fixed point can be reached after countably many iterations of $f_{\varphi, V}$. [48] gave a syntactic description of all continuous fixed point formulas that form a sub-fragment of all constructive formulas. Using this description it is easy to see that $C \varphi$ is in the continuous and hence in the constructive fragment of all fixed point formulas. Therefore, in order to compute common knowledge we need only countably infinite iterations.

It is easy to see that $C \varphi$ expresses the reflexive transitive closure, i.e., 'some $\varphi$-world is reachable in finitely many $\sim_{E}$-steps' ([11, Example 6]). Next we will compute common knowledge following our fixed point definition in some of the models shown in Figure 2 In $M_{1}$ we have $V(p)=\left\{s_{1}\right\}$. So if $\varphi=p \wedge \square_{a} x \wedge \square_{b} x$, then

$$
\begin{aligned}
f_{\varphi, V}^{0}(S) & =\llbracket \varphi \rrbracket]_{V_{x}^{S}} \\
& \left.=V(p) \cap \llbracket \square_{a} x \rrbracket\right]_{V_{x}^{S}} \cap \llbracket\left[\square_{b} x\right]_{V_{x}^{S}} \\
& =\left\{s_{1}\right\} \cap S \cap S=\left\{s_{1}\right\} .
\end{aligned}
$$

Then

$$
\begin{aligned}
f_{\varphi, V}^{1}(S) & \left.\left.=\left\{s_{1}\right\} \cap \llbracket\left[\square_{a} x\right]\right]_{V_{x}^{f_{\varphi, V}^{0}(S)}} \cap\left[\llbracket \square_{b} x\right]\right]_{V_{x} f_{\varphi, V}^{0}(S)} \\
& \left.\left.=\left\{s_{1}\right\} \cap \llbracket\left[\square_{a} x\right]\right]_{\left.V_{x}^{\prime} s_{1}\right\}} \cap \llbracket\left[\square_{a} x\right]\right]_{\left.V_{x}^{\left\{s_{1}\right.}\right\}} \\
& =\left\{s_{1}\right\} \cap \emptyset \cap \emptyset=\emptyset .
\end{aligned}
$$

Finally, observe that $f_{\varphi, V}(\emptyset)=\emptyset$. So we reached the least fixed point and $[\llbracket C p]=$ $[\llbracket v \times \varphi]=\emptyset$.

Now consider the second model and the formula $\sigma=\neg K_{a} p \wedge \neg K_{b} p$. It is easy to see that in $M_{2}$ we have $[\sigma \sigma]_{V}=S$. Let $\varphi=\sigma \wedge \square_{a} x \wedge \square_{b} x$. Then $f_{\varphi, V}^{0}(S)=\llbracket \varphi \rrbracket_{V_{x}^{S}}=$ $\llbracket \sigma \rrbracket_{V} \cap\left[\square_{a} x \rrbracket_{V_{x}^{S}} \cap \llbracket \square_{b} \rrbracket_{V_{x}^{S}}=S \cap S \cap S=S\right.$. This means that $S$ is the greatest fixed point of $f_{\varphi, V}$. So $[[C \sigma]=\llbracket[v x \varphi]]=S$. We leave it up to the reader to compute common knowledge of various formulas in other models depicted in Figure 2.

Note that $M_{1}, s_{1} \models E \varphi \leftrightarrow C \varphi$, but also that $M_{4}, s_{4} \models E \neg K_{b} p \wedge \neg C \neg K_{b} p$. The pointed epistemic model $M_{2}, s_{2}$ not only models the scenario $\sigma: p \wedge \neg K_{a} p \wedge \neg K_{b} p$ but also that this is common knowledge: $M_{2}, s_{2} \models C \sigma$. It is the only pointed model $M_{i}, s_{i}(i \leq 5)$ with this property.

Correspondence properties make modal logic a flexible tool to model epistemic and doxastic logics: once one has decided on the desired properties of the informational attitude, like negative introspection, the Kripke models obtained need just to satisfy an additional property, like Euclideaness. It also helps provide a neat analysis of informational group notions. There are also some drawbacks using Kripke models for knowledge and belief: we will come back to this in Section 4.1. 


\subsection{Completeness}

In this section we briefly recall soundness and completeness of some important modal logics. Let $L$ be a (normal) modal logic defined in Section 2. Recall that a (normal) modal logic $L$ is called sound wrt a class $\mathrm{K}$ of Kripke frames if $F \models L$ for each $F \in \mathrm{K}$. Logic $L$ is called complete wrt $\mathrm{K}$ if for each formula $\varphi$, if $\varphi$ is $L$-consistent (i.e., $L \cup\{\varphi\} \forall \perp$ ), then there is $F \in \mathrm{K}$ such that $\varphi$ is satisfied in $F$. A frame $F$ is called an $L$-frame if $F \models L$. It is easy to see that if $L$ is sound and complete wrt some class $\mathrm{K}$, then it is sound and complete wrt the class of all $L$ frames. $L$ is called strongly complete wrt a class $\mathrm{K}$ of Kripke frames if for each set of formulas $\Gamma$, if $\Gamma$ is $L$-consistent (i.e., $L \cup \Gamma \forall \perp$ ), then there is $F \in \mathrm{K}$ such that $\Gamma$ is satisfied in $F$.

Recall also that a transitive frame $F=\langle S, R\rangle$, is called rooted if there exists $s \in S$, called a root, such that for each $s^{\prime} \in S$ with $s^{\prime} \neq s$ we have $s R s^{\prime}$. It is well known that if a logic is sound a complete, then it is sound and complete wrt a class of rooted $L$-frames.

A standard method for proving completeness of modal logics is via the canonical model construction. We briefly review it here. In the next section we explain how this construction is generalised to the topological setting. All the details can be found in any modal logic textbook, e.g., [32] or [36].

Given a logic $L$, one considers the set $S^{C}$ of all maximal $L$-consistent sets of formulas. A relation $R^{C}$ on $S^{C}$ is defined in the following way: for each $\Gamma, \Delta \in S^{C}$, $\Gamma R_{\square}^{C} \Delta$ if for each formula $\varphi$ we have $\square \varphi \in \Gamma$ implies $\varphi \in \Delta$. Finally, the valuation $V^{C}$ on $S^{C}$ is defined by $\Gamma \in V^{C}(p)$ if $p \in \Gamma$. The model $M^{C}=\left\langle S^{C}, R^{C}, V^{C}\right\rangle$ is called the canonical model of $L$. Then one proves the Truth Lemma stating that for each formula $\varphi$ and $\Gamma \in S^{C}$ :

$$
M^{C}, \Gamma \models \varphi \text { iff } \varphi \in \Gamma .
$$

Now suppose $\varphi$ is $L$-consistent. Then by the Lindenbaum Lemma (see, e.g., [32], [36]), $\{\varphi\}$ can be extended to a maximal consistent set $\Gamma$. By the Truth Lemma, $M^{\mathcal{C}}, \Gamma \models \varphi$. Thus, we found a frame $\left\langle S^{C}, R^{C}\right\rangle$ that satisfies $\varphi$. In order to finish the proof we need to show that $\left\langle S^{C}, R^{C}\right\rangle$ is an $L$-frame. If the latter is satisfied, then $L$ is called canonical. Therefore, canonical modal logics are Kripke complete.

It is a classical result of modal logic that if a normal modal logic $L$ is axiomatised by Sahlqvist formulas, then $L$ is canonical, and hence Kripke complete, see e.g., [32] or [36]. Together with the Sahlqvist-van Benthem correspondence result discussed in the previous section, this theorem guarantees that every logic axiomatised by Sahlqvist formulas is sound and complete wrt a first-order definable class of Kripke frames. As a result we obtain that epistemic and doxastic logics $S 4_{m}, S 5_{m}, K D 45_{m}$ are all sound and complete with respect to corresponding classes of Kripke frames discussed in the previous section.

We now summarise a number of completeness results for epistemic logics in the following theorem. Proofs and extensions of them can be found in [46, Chapter 3.1], and [86, Chapter 2] for epistemic logics, in [86, Chapter 1] and [33, Chapter 4] for normal modal logics in general and in [50] for $L T L$. The set of models $\mathcal{L} \mathcal{I}$ is 
the set of all linear orders: think of them as $M=\langle\mathbb{N}$, Succ, $V\rangle$, where $x$ Succ $y$ iff $y=x+1$.

Theorem 2. In the following, $m \geq 1$. Item 6 presents a logic and a semantics to which it is sound and complete. All the other items present logics that are strongly sound and complete with respect to the mentioned semantics:

$$
\begin{array}{ll}
1 K_{m} \text { and } \mathcal{K}_{m} & 5 S 5_{m} \text { and } \mathcal{S} 5_{m} \\
2 S 4_{m} \text { and } \mathcal{S} 4_{m} & 6 S 5_{m}^{C} \text { and } \mathcal{S} 5_{m}^{C} \\
3 K D 45_{m} \text { and } \mathcal{K D} 45_{m} & 7 S 5_{m}^{D} \text { and } \mathcal{S} 5_{m}^{D} \\
4 S 5_{1} \text { and } \mathcal{U}_{1} & 8 L T L \text { and } \mathcal{L I N}
\end{array}
$$

Note that by (2), when only finite formulas are allowed, we will not be able to find a strong completeness result for common knowledge: the set $\{E p, E E p, \ldots\} \cup$ $\{\neg C p\}$ is consistent, but not satisfiable. For logics with distributed knowledge, we saw that in the canonical model, we only have $R_{D}^{C} \subseteq \cap_{a \in \mathrm{Ag}} R_{a}^{C}$. To also obtain the converse, for any two sets $\Gamma$ and $\Delta$ for which we have $\Gamma\left(\cap_{a \in \mathrm{Ag}} R_{a}^{C}\right) \Delta$, but not $\Gamma R_{D}^{C} \Delta$, one can replace $\Delta$ by $n$ copies $\Delta_{1}, \ldots, \Delta_{n}$, with $\Gamma R_{i}^{C} \Delta_{i}$. Of course, in the context of for instance $S 5$, one needs to take care that the relations remain an equivalence relation, but this can be done: for a discussion see for instance [71].

\subsection{Expressivity and definability of Epistemic Models}

Speaking with van Benthem's [13, p. 32], one can ask: 'When are two information models the same?' For instance, although all our five pointed models $M_{i}, s_{i}$ verify the same scenario $\sigma$, do they differ in some other sense?

Definition 3 ((Bi-)simulation). Let $M=\langle S, \sim, V\rangle$ and $M^{\prime}=\left\langle S^{\prime} \sim^{\prime}, V^{\prime}\right\rangle$ be two epistemic models. A simulation between $M$ and $M^{\prime}$ is a relation $R \subseteq S \times S^{\prime}$ such that

Harmony If $s R s^{\prime}$ then for all $p \in$ At, $s \in V(p)$ iff $s^{\prime} \in V^{\prime}(p)$

Forth For all $a \in \mathrm{Ag}$, if $s \sim_{a} t$ and $s R s^{\prime}$, then for some $t^{\prime} \in S^{\prime}, t R t^{\prime}$ and $s^{\prime} \sim_{a}^{\prime} t^{\prime}$

$R$ is called a bisimulation if it moreover satisfies

Back For all $a \in \mathrm{Ag}$, if $s^{\prime} \sim_{a}^{\prime} t^{\prime}$ and $s R s^{\prime}$, then for some $t \in S, t R t^{\prime}$ and $s \sim_{a} t$

If $s R s^{\prime}$ and $R$ is a simulation, we say that $M, s$ simulates $M^{\prime}, s^{\prime}$; if $R$ is a bisimulation, we say that $M, s$ and $M^{\prime}, s^{\prime}$ are bisimilar.

As an example, note that $M_{1}, s_{1}$ simulates $M_{3}, s_{3}$, while $M_{1}, s_{1}$ and $M_{5}, s_{5}$ are bisimilar. Roughly speaking, if $M, s$ simulates $M^{\prime}, s^{\prime}$, then ignorance (i.e., an $M_{a^{-}}$ formula) is preserved from $M, s$ to $M^{\prime}, s^{\prime}$, and knowledge is preserved in the other direction. A bisimulation preserves both.

Lemma 1. [13, Invariance Lemma] Let $M$ and $M^{\prime}$ be finite models. Let $\mathrm{L}=\mathrm{L}(\mathrm{At}$, $\mathrm{Op}, \mathrm{Ag})$, with $\mathrm{Op}=\left\{K_{a} \mid a \in \mathrm{Ag}\right\}$. Then the following are equivalent: 
(a) $M, s$ and $M^{\prime}, s^{\prime}$ are bisimilar,

(b) $M$, s and $M^{\prime}, s^{\prime}$ satisfy the same formulas $\varphi \in \mathrm{L}$.

Proof. For (a) $\Rightarrow$ (b) one can follow a standard argument using induction on $\varphi$. For the converse, let (b) be given and define $x R x^{\prime}$ as $x$ and $x^{\prime}$ satisfy the same formulas from L. Clearly Atoms holds for $R$, and also, $s R s^{\prime}$. To show Forth, suppose $x R x^{\prime}$ while $x \sim_{a} y$ for some agent $a$. Suppose there is no state $y^{\prime}$ in $S^{\prime}$ with $y \sim_{a} y^{\prime}$ for which $y R y^{\prime}$ holds, i.e., for every $y^{\prime}$ with $y \sim_{a} y^{\prime}$ there is a formula $\chi_{y^{\prime}-}^{x+}$ true in $x$, but false in $y^{\prime}$. Let $\chi$ be $\bigwedge_{\left\{y^{\prime} \mid y \sim a y^{\prime}\right\}} \chi_{y^{\prime}-}^{x+}$, then $M, x=\chi$ while $M^{\prime}, x^{\prime}=\neg \chi$, contradicting $x R x^{\prime}$. Back is proven similarly.

This lemma implies that our pointed models $M_{1}, s_{1}$ and $M_{2}, s_{2}$ are not bisimilar, since $M_{a} K_{b} \neg p$ is true in the first, but not in the second.

Where the invariance lemma says that 'bisimulation has exactly the expressive power of the modal language' $([9$, p. 56]) the following State Definition Lemma says that every pointed epistemic model can be characterised by an epistemic formula in the language with common knowledge.

Lemma 2. [13, State Definition Lemma] For each finite pointed epistemic model $M$, s there is a formula $\varphi \in \mathrm{L}(\mathrm{At}, \mathrm{Op}, \mathrm{Ag})$, with $\mathrm{Op}=\left\{K_{a} \mid a \in \mathrm{Ag}\right\} \cup\{C\}$ such that the following are equivalent (where $M^{\prime}$ is finite):

(a) $M^{\prime}, s^{\prime}=\varphi$,

(b) $M, s$ is bisimilar to $M^{\prime}, s^{\prime}$.

The conditions in both lemmas are necessary: finite epistemic states are not definable up to simulation in the language with common knowledge, nor is bisimulation to finite epistemic models definable in the language without common knowledge ([39]).

For later reference, we conclude this section by stating van Benthem's characterisation theorem for modal logic. The standard translation $S T_{x}$ takes a modal formula and returns a first-order formula using the clauses $S T_{x}(p)=P x$, it commutes with the Boolean connectives and stipulates that $S T_{x}(\square \varphi)=\forall y\left(x R_{\square} y \Rightarrow S T_{y}(\varphi)\right)$.

Theorem 3 ([24]). The following are equivalent for first-order formulas $\Phi(x)$ :

1. $\Phi(x)$ is invariant under bisimulation,

2. $\Phi(x)$ is equivalent to $S T_{x}(\varphi)$ for some modal formula $\varphi$.

\subsection{Epistemic Temporal Frames}

Van Benthem and Pacuit ([21]) formalise a notion of time using so-called epistemic temporal frames $\mathcal{F}=\langle\Sigma, \mathcal{H}, \sim\rangle$, where $\Sigma$ is a set of events (say, possible moves in a game) and $\mathcal{H}$ is a set of histories. For this chapter, $\mathcal{F}$ can be thought of as a finitely branching rooted tree labeled with events. The histories are then nothing else than 
strings of events. Figure 3 (left) provides an example. Frame $\mathcal{F}$ in this figure denotes a game where agent $a$ can first decide to move $L$ or $R$, after which $b$ can move either $l$ or $r$ (so $\Sigma=\{L, R, l, r\}$ ). For epistemic temporal frames, the indistinguishability relation is defined over histories, in $\mathcal{F}$ of Figure 3 for instance, we have $L \sim_{b} R$ (agent $b$ does not know which move $a$ starts with) and $L l \sim_{a} R l$ (if $b$ plays $l$, agent $a$ forgets what his own initial move has been).

The semantic counterpart of no surprise (NS) would then say that for all finite histories $H, H^{\prime} \in \mathcal{H}$ and all events $e \in \Sigma$ with $H e, H^{\prime} e \in \mathcal{H}$, if $H \sim_{a} H^{\prime}$, then $H e \sim_{a} H^{\prime} e$. The converse of this would guarantee PR. One might be tempted to think that this converse ensures $K_{a} \bigcirc \varphi \rightarrow \bigcirc K_{a} \varphi$, but this is not the case for $\varphi$ that refer to what is the case now (like, 'it is $3 \mathrm{am}$ ') or that refer to ignorance (like ' $a$ does not know that $\psi^{\prime}$ '), knowledge of such properties may be given up, even (or especially when) provided with more information (see [46, page 130] for further discussion). A bounded agent does not have perfect recall, but instead has a finite bound on the number of preceding events which they can remember. [21] calls an agent synchronised if $H \sim_{a} H^{\prime}$ can only occur for histories $H$ and $H^{\prime}$ that have the same length (so the agent would know how many moves have been played, or, more generally, know the time of the global clock). In $\mathcal{F}$ of Figure 3, both agents are synchronised, agent $b$ does not satisfy no surprise (he cannot distinguish the histories $L$ and $R$, but if in both the same action (say $l$ ) is performed, he can distinguish the result), while agent $a$ does not satisfy perfect recall: he cannot distinguish $L l$ and $R l$, although he knew the difference between $L$ and $R$.
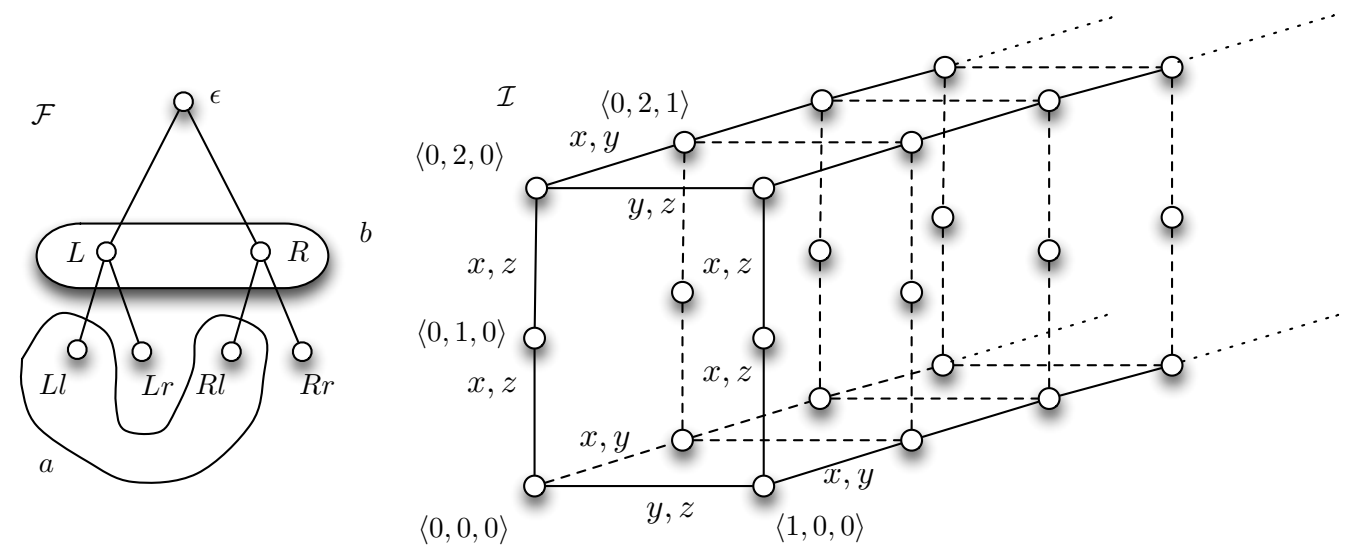

Fig. 3 An epistemic temporal frame $\mathcal{F}$ (left) and an interpreted system $\mathcal{I}$ (right).

Following the pioneering [58] of Halpern and Vardi on the complexity of reasoning about knowledge and time, van Benthem and Pacuit highlight in [21] how several choices in the formalism can have quite dramatic consequences for the decidability and computational complexity (of the validity problem) of the underlying logic. Choices that heavily influence the complexity regard for instance the language 
(does it include an operator for common knowledge, do we allow for temporal operators for the past and for the future?), structural conditions on the underlying event structure (what if we give up some conditions of an epistemic temporal frame, or look at forests rather than trees?) and conditions on the reasoning abilities of the agents (perfect recall, no surprise, synchronisation, bounded agents). Moreover, [21] marks the start of a research paradigm that compares and links existing approaches to epistemic logic (Kripke models, interpreted systems ([46])), and 'Parikh style' logic ([92]), time (history based structures ([92]), runs ([46])), and dynamics, including PDL-style logic ([61]) and dynamic epistemic logic (see Chapter 7). Van Benthem further helped clarify the link between interpreted systems, epistemic temporal logic and dynamic epistemic logic in [19].

Chapter in this volume by Goranko and Pacuit presents a more comprehensive survey of temporal epistemic frameworks. For examples of completeness results regarding systems for knowledge and time, we refer to Theorem 4. For a general discussion on completeness and complexity issues for such logics, and further references, we refer to van Benthem and Pacuit's [21].

\subsection{Interpreted Systems}

In the 1980s, computer scientists became interested in epistemic logic. This line of research flourished in particular by a stream of publications around Fagin, Halpern, Moses and Vardi. Their important textbook [46] surveys their work on epistemic logic over a period of more than ten years. The emphasis in this work is on interpreted systems (IS) as an underlying model for their framework, a semantics that also facilitates reasoning about knowledge during computation runs in a natural way. The key idea behind IS is two-fold:

- It provides for a so-called grounded semantics of epistemic logic;

- It adds a dynamic and computational component to this through the notions of run and protocol.

Where in an epistemic model the equivalence relations $\sim_{a}$ are given, in an interpreted system they are grounded in the notion of observational equivalence. To be more precise (for formal definitions we refer to [46]), let $L_{a}$ be a set of possible local states for agent $a$. For example, when modeling a distributed computation, such a local state could provide the value of the variables associated with processor $a$, or in a card game it could be the enumeration of cards held by player $a$. Moreover, let $L_{e}$ be a set of possible states for the environment. This state could have information about a global clock, or keep track of whose turn it is in a card game. The set of global states of an interpreted system with $m$ agents is then $\mathcal{G}=L_{e} \times L_{1} \times \ldots \times L_{m}$. If $\mathbf{s}=\left\langle s_{e}, s_{1}, \ldots, s_{m}\right\rangle \in \mathcal{G}$ with $\mathbf{s}_{a}$ we mean $s_{a}(a \in\{e\} \cup \mathrm{Ag})$. An example of an interpreted system is $\mathcal{I}$ of Figure 3, where the environment is not modelled (it is constant, say), $L_{x}=\{0,1\}, L_{y}=\{0,1,2\}$ and $L_{z}=\mathbb{N}$. 
Two global states $\mathbf{s}=\left\langle s_{e}, s_{1}, \ldots, s_{m}\right\rangle$ and $\mathbf{s}^{\prime}=\left\langle s_{e}^{\prime}, s_{1}^{\prime}, \ldots, s_{m}^{\prime}\right\rangle$ are now defined to be indistinguishable for agent $i$, written $\mathbf{s} \sim_{i}^{I} \mathbf{s}^{\prime}$, if $i$ 's local state is the same in both, i.e., if $s_{i}=s_{i}^{\prime}$. This is clearly an equivalence relation, and hence this notion of interpreted system gives rise to knowledge of veridical and introspective agents. In the most general case, we will not always consider the full cartesian product $\mathcal{G}$ but some subset $\mathcal{J} \subseteq \mathcal{G}$ of it. This represents situations where overall constraints of the system prevent some global states from being part of the model.

A run over $\mathcal{J} \subseteq \mathcal{G}$ is a function $r: \mathbb{N} \rightarrow \mathcal{J}$. Intuitively, this captures a computation, or a behaviour of the system. If $r(m)=\mathbf{s}$, then $r_{i}(m)=\mathbf{s}_{i}$ is the local state of agent $i$ in run $r$ at time $m$. A pair $(r, m)$ is called a point. An interpreted system $\mathcal{I}$ is a pair $\left\langle\mathcal{R}, V^{I}\right\rangle$, where $\mathcal{R}$ is a set of runs and $V^{I}(p)$ denotes for each propositional variable $p$ (' $x$ is 3', or ' $i$ holds card diamond 9') the set of global states in which it is true. In other words, we assume that the truth of atoms does not depend on 'where we are in the run', but only on the global state (in particular, if a run $r$ visits the same global state twice, i.e., $r(m)=r(m+k)$, for some $m, k \in n$, then the truth of atoms is the same in both points). Moreover, to quote [46, page 112], 'Quite often, in fact, the truth of a primitive proposition q of interest depends, not on the whole global state, but only on the component of some particular agent'. In such cases, the valuation $V^{I}(q)$ respects the locality of $q$, which means that, if $\mathbf{s} \sim_{i} \mathbf{s}^{\prime}$, then $\mathbf{s} \in V^{I}(q)$ iff $\mathbf{s}^{\prime} \in V^{I}(q)$. In such a case, the fact that $i$ knows the truth of such a property is common knowledge. To be more precise, suppose that there is a property $x_{i}=0$, which is true exactly when in $i$ 's local state, the variable $x_{i}$ is equal to 0 . Then, we have $C\left(x_{i}=0 \rightarrow K_{i}\left(x_{i}=0\right)\right)$.

It is easy to see that an interpreted system $\mathcal{I}=\left\langle\mathcal{R}, V^{I}\right\rangle$ gives rise to an epistemic model $M_{I}=\langle S, \sim, V\rangle$, by taking for $S$ all the points generated by $\mathcal{R}$, and where $(r, m) \sim_{i}\left(r^{\prime}, m^{\prime}\right)$ iff $r(m) \sim_{i}^{I} r^{\prime}\left(m^{\prime}\right)$ and $(r, m) \in V(p)$ iff $r(m) \in V^{I}(p)$ (so, $\sim$ and $V$ defined over points $(r, m)$ is determined by $\sim^{I}$ and $V^{I}$ on global states $r(m)$ ).

If we now define $\mathcal{I}, r, m \models \varphi$ as $M_{I}, r(m) \models \varphi$, we have an interpretation for the individual and group epistemic notions discussed in Section 2.1. For full interpreted systems, where $\mathcal{G}$ is the full cartesian product $L_{e} \times L_{1} \times \cdots \times L_{m}$, we have that common knowledge is constant over all runs. This is so since for every two global states $\mathbf{s}=\left\langle s_{e}, s_{1}, \ldots, s_{m}\right\rangle$ and $\mathbf{s}^{\prime}=\left\langle s_{e}^{\prime}, s_{1}^{\prime}, \ldots, s_{m}^{\prime}\right\rangle$ there is a third state $\mathbf{t}=\left\langle s_{e}, s_{1}, s_{2}^{\prime}, \ldots\right\rangle$ 'epistemically connecting them'. The notion of a run in an interpreted system also directly facilitates the interpretation of temporal formulas: we define for instance $\mathcal{I}, r, m \models \bigcirc \varphi$ as $\mathcal{I}, r, m+1 \models \varphi$.

For our example system $\mathcal{I}$ we assume to have propositional atoms like $x=0$, $z=9$. We also identify three runs, $r_{0}, r_{1}$ and $r_{2}$. In all of them, the variable $z$ is increased by 1 in each step, where $z=0$ in $\left(r_{0}, 0\right)$ and $\left(r_{2}, 0\right)$ and $z=1$ in $\left(r_{1}, 0\right)$. In both $r_{0}$ and $r_{2}$, the values of $\langle x, y\rangle$ are a clockwise walk through the $x y$ plane: $\langle x, y\rangle$ $=\langle 0,0\rangle,\langle 0,1\rangle,\langle 0,2\rangle,\langle 1,2\rangle,\langle 1,1\rangle,\langle 1,0\rangle,\langle 0,0\rangle, \ldots$ In $r_{2}$, the variables $x$ and $y$ are both 0 at even places, and both 1 at odd places.

$$
\begin{aligned}
& r_{0}:\langle 0,0,0\rangle\langle 0,1,1\rangle\langle 0,2,2\rangle\langle 1,2,3\rangle\langle 1,1,4\rangle\langle 1,0,5\rangle\langle 0,0,6\rangle \ldots \\
& r_{1}:\langle 0,0,1\rangle\langle 0,1,2\rangle\langle 0,2,3\rangle\langle 1,2,4\rangle\langle 1,1,5\rangle\langle 1,0,6\rangle\langle 0,0,7\rangle \ldots \\
& r_{2}:\langle 0,0,0\rangle\langle 1,1,1\rangle\langle 0,0,2\rangle\langle 1,1,3\rangle\langle 0,0,4\rangle\langle 1,1,4\rangle\langle 0,0,4\rangle \ldots
\end{aligned}
$$


Let $\mathcal{I}_{01}$ consist of the runs $r_{0}$ and $r_{1}$ whereas $\mathcal{I}_{12}$ has the runs $r_{1}$ and $r_{2}$. We then have, in $\mathcal{I}_{02}, r,\langle 0,0,0\rangle$ :

$$
K_{x} x=0 \wedge \neg K_{x} y=0 \wedge E \square\left(x=0 \leftrightarrow K_{x} x=0\right) \wedge \neg K_{x} \bigcirc x=1 \wedge K_{z} \bigcirc z=1
$$

In order to semantically characterise perfect recall in an interpreted system, let, for an agent $i$, his local-state sequence at the point $(r, m)$ be the sequence of local states he has seen in run $r$ up to time $m$, without consecutive repetitions. So, for the run $r_{0}$ above, the local state sequence for agent $x$ at time 4 equals $\langle 01\rangle$, for agent $y$ it is $\langle 0,1,2,1\rangle$, and for $z$ it is $\langle 0,1,2,3,4\rangle$. We now say that $i$ has perfect recall $p r$ if whenever $(r, m) \sim_{i}\left(r^{\prime}, m^{\prime}\right)$, then $i$ has the same local-state sequence at $(r, m)$ and $\left(r^{\prime}, m^{\prime}\right)$. In the system $\mathcal{I}_{02}$, agent $z$ has perfect recall, but in $\mathcal{I}_{01}$, he has not. To see the latter, we have $\left(r_{0}, 1\right)=\langle 0,1,1\rangle \sim_{z}\langle 0,0,1\rangle=\left(r_{1}, 0\right)$, whereas the state sequence for $z$ in $\left(r_{0}, 1\right)$ is $\langle 01\rangle$ while in $\left(r_{1}, 0\right)$ it is $\langle 1\rangle$. Indeed, it is easy to see that we have $\mathcal{I}_{01}, r_{0},\langle 0,0,0\rangle \models K_{z} \bigcirc y=1 \wedge \neg \bigcirc K_{z} y=1$

An interpreted system $\mathcal{I}=\left\langle\mathcal{R}, V^{I}\right\rangle$ satisfies sync if agents know what time it is, i.e., if for all agents $i$, we have that $(r, m) \sim_{i}\left(r^{\prime}, m^{\prime}\right)$ implies $m=m^{\prime}$.

Theorem 4. We have the following (see [46, Chapter 8]).

1. Both $S 5_{m}+L T L$ and $S 5_{m}^{C}$ are sound and complete with respect to the set of all interpreted systems $\mathcal{I} \mathcal{N} \mathcal{T}_{m}$ for $m$ agents.

2. Both $S 5_{m}+L T L$ and $S 5_{m}^{C}$ are sound and complete with respect to the set of synchronised interpreted systems $\mathcal{I} \mathcal{N} \mathcal{T}_{m}^{\text {sync }}$.

3. $S 5_{m}+L T L+\mathbf{P R}$ is sound and complete with respect to the set of synchronised interpreted systems with perfect recall $\mathcal{I} \mathcal{N} \mathcal{T}_{m}^{\text {sync,pr }}$.

The first item of Theorem 4 suggests that the the static, non-temporal validities of interpreted systems are axiomatised by $S 5_{m}$, and hence that interpreted systems are in some sense equivalent to Kripke models. This idea was taken up by Lomuscio and Ryan in e.g., [80], roughly (the analysis in [80] is appropriately done at the level of frames, we give here a summary on the level of models) as follows. In order to link interpreted systems with $\mathcal{S} 5_{m}$ structures, [80] restricts themselves to structures (1) without dynamic component (i.e., systems without runs), (2) where the state space is the full cartesian product $\mathcal{G}$ and (3) where the environment is not modelled in a global state. This leads to a notion of hypercube, which is just $L_{1} \times \cdots \times L_{2}$, where $L_{i}$ is as before, as is the agents' accessibility relation. Call the set of hypercubes for $m$ agents $\mathcal{H}_{m}$. From what we have said above, it follows that in a hypercube $H$, common knowledge is constant, i.e., for all states $\mathbf{s}$ and $\mathbf{t}$, we have $H, \mathbf{s} \models C \varphi$ iff $H, \mathbf{t}=C \varphi$ (if $R_{C} \mathbf{s u}$, then $R_{1}\left\langle t_{1}, t_{2}, \ldots, t_{m}\right\rangle\left\langle t_{1}, u_{2}, \ldots u_{m}\right\rangle$ and $R_{2}\left\langle t_{1}, u_{2}, \ldots u_{m}\right\rangle\left\langle u_{1}, u_{2}, \ldots u_{m}\right\rangle$, hence $\left.R_{C} \mathbf{t u}\right)$. However, to show that this discriminates the validities in $\mathcal{H}_{m}$ from those in $\mathcal{S} 5_{m}$, one would need a universal modality. But we also have the following, which shows that hypercubes behave different form $\mathcal{S} 5_{m}$ models (recall that distributed knowledge $D \varphi$ is true in a state $s$ if $\varphi$ holds in all $t$ for which $s R_{D} t$, where $R_{D}=\sim_{1} \cap \ldots \cap \sim_{m}$ ):

Observation 1 Let $H \in \mathcal{H}$. Let $i, j \in$ Ag. Recall that $M_{i} \varphi=\neg K_{i} \neg \varphi$. 
1 In $H$, we have $R_{D}$ is the identity, that is, $\mathbf{s} R_{D} \mathbf{t}$ iff $\mathbf{s}=\mathbf{t}$.

2 For all global sates $\mathbf{s}^{1}, \ldots, \mathbf{s}^{m}$, there is a global state $\mathbf{s}$ with $\mathbf{S} \sim_{i} \mathbf{s}^{i}$, for all $i \leq m$.

From these semantic properties, we derive the following validities on hypercubes:

$3 \mathcal{H}_{m}=\varphi \leftrightarrow D \varphi$

$4 \mathcal{H}_{m}=M_{i} K_{j} \varphi \rightarrow K_{j} M_{i} \varphi$

However, those validities do not transfer to $\mathcal{S} 5_{M}$ :

$5 \mathcal{S} 5_{m} \not \models \varphi \leftrightarrow D \varphi$

$6 \mathcal{S} 5_{m} \not \forall M_{i} K_{j} \varphi \rightarrow K_{j} M_{i} \varphi$

Proof. Item 1 follows from the fact that $\mathbf{s}=\left\langle s_{1}, \ldots, s_{m}\right\rangle R_{D}\left\langle t_{1}, \ldots t_{m}\right\rangle=\mathbf{t}$ iff $s_{1}=$ $t_{1} \& \ldots \& s_{m}=t_{m}$ iff $\mathbf{s}=\mathbf{t}$. This immediately implies item 3 . For item 5 , observe that in $M_{2}, s_{2}$ of Figure 2 it holds that $p \wedge \neg D p$. For item 2 , take $\mathbf{s}=\left\langle\mathbf{s}_{1}^{1}, \mathbf{s}_{2}^{2}, \ldots, \mathbf{s}_{m}^{m}\right\rangle$ (i.e., take agent 1's local state from $\mathbf{s}^{1}$, agent 2 's local state from $\mathbf{s}^{2}$, etc). Obviously, $\mathbf{s} \sim_{i} \mathbf{s}^{i}$. One can use a correspondence theory argument to show that this implies item 4 (see e.g., [80, Lemma 9]). For item 6, consider the model $M_{1}$ in Figure 1. We extend this model to $M_{1}^{\prime}$ as follows: it makes $q$ true in the two right-most states. Then we have $M_{1}^{\prime}, s_{1} \models M_{a} K_{b} \neg q \wedge M_{b} K_{a} q$, in other words, $M_{1}^{\prime} \not \models \neg K_{a} \neg K_{b} \neg q \rightarrow K_{b} M_{a} \neg q$.

Observation 1 implies that hypercubes, the static part of interpreted systems, are a special kind of $\mathcal{S} 5_{m}$ models, which verify some additional properties. In fact, the following theorem (for which proof we refer to that of [80, Theorem 20]) shows that Observation 1 in fact sums up everything that separates $\mathcal{H}_{m}$ from $\mathcal{S} 5_{m}$ :

Theorem 5 (Based on Theorem 20 of [80]). Let $\mathcal{H S} 5_{m} \subset \mathcal{S} 5_{m}$ be the set of $\mathcal{S} 5_{m}$ models $M=\langle S, \sim, V\rangle$ that satisfy:

1. $\forall s t \in S s\left(\sim_{1} \cap \cdots \cap \sim_{m}\right) t$ iff $s=t$

2. $\forall s_{1}, \ldots, s_{m} \in S \exists s \in S$ such that $\forall i \in \operatorname{Ag} s \sim_{i} s_{i}$.

Then the validities (of the language with operators $K_{i}, C$ and $D$ ) in $\mathcal{H S} 5_{m}$ and $\mathcal{H}_{m}$ are the same.

So, the grounded semantics for knowledge, where it is explained where the accessibility relations come from, when implemented through hypercubes, the static counterpart of full interpreted systems, has as a consequence that we get the two additional properties 3 and 4 of Observation 1 for knowledge, as compared to $\mathcal{S} 5_{m}$. Of course, on can give up the condition of full interpreted systems (in which case property 4 would disappear), or think about different ways of groundedness in the first place.

Many theories of multi-agent systems, which try to model notions like knowledge, belief, intentions, commitments, obligations and actions of agents are embedded in the philosophical brand of modal logic, in a way that is similar to what we discuss here for the knowledge of agents. Computational groundedness was put forward (cf. [104]) to make such theories more relevant to practitioners in multi-agent systems and distributed artificial intelligence in general. It is therefore no surprise 
that attempts to make such intentional notions (see also Chapter 12 of this volume) grounded are not limited to the notion of knowledge only. For instance, Su and others ([99]) provided a grounded model for the notions of knowledge, belief and certainty. Roughly, a state in their models has an external and an internal part: the external part determines what of the system is visible, and what is not visible, while the internal part specifies for each agent his perception of the visible part of the environment state and the plausible invisible parts of the invisible part of the environment stat that the agent thinks possible. Lomuscio and Sergot even use the notion of interpreted system to show "how it can be trivially adapted to provide a basic grounded formalism for some deontic issues' ([81, page 3]). Their models are basically hypercubes, where each local state $L_{i}$ is then partitioned in a set of green states (allowed states of computation) and red states (disallowed states). This enables them to define a notion $\mathcal{O}_{i} \varphi$, with the meaning that 'in all the possible correctly functioning alternatives of agent $i, \varphi$ is the case'.

\section{Generalised Structures for Knowledge}

Kripke structures provide a very natural way to model uncertainty and (lack of) information, and they are conceptually relatively easy. Depending on the kind of uncertainty one wants to model, one can often employ correspondence theory and, in a modular way, add additional constraints on the agents' accessibility relations. But there is also a criticism using this semantics, going in the other direction even if we do not impose any additional constraints on those relations, do we not get properties (of knowledge or belief) that are in fact too strong? This problem is known as the logical omniscience problem, and neighbourhood semantics is developed partially with the aim to address this. Finally, there is a stream of topological models for epistemic languages, which have their own virtues.

\subsection{Neighbourhood Semantics}

So what are possible shortcomings of using relational models for knowledge and belief? First of all, although this is not implied by the semantics, it is almost always assumed that all agents are equal: their knowledge and beliefs all satisfy the same properties. Indeed, in $S 5_{m}$ we have, for all $\varphi$, that $\vdash K_{a} \varphi$ iff $\vdash \varphi$ iff $\vdash K_{b} \varphi$. Van Benthem and Liu are among the first to take seriously that 'epistemic agents may have different powers of observation and reasoning' ([20]), and allow for a 'diversity of logical agents'. Secondly, if one wants to express that the beliefs of agent $a$ are correct by adding the axiom $B_{a} \varphi \rightarrow \varphi$ to a logical system, this property becomes globally valid: every agent knows it, it would even become common knowledge, and in a temporal setting it will hold forever. A first step to address this was made in [40]. 
A more fundamental criticism against using normal modal logic to model information of agents is known as logical omniscience. No agent is a perfect reasoner, so no agent will know all tautologies (of $S 5$, or even the weakest normal modal logic $K$ ). This observation questions the intuitive soundness of Nec. Indeed, security protocols for communication or authentication that use cryptographic keys are based on the assumption that agents are not able to oversee all the consequences of the underlying theory (like inferring whether a given number is prime).

A similar criticism is sometimes used against axiom $\mathbf{K}$ : whereas an agent applying $\mathbf{K}$ once seems rather innocent, having it as an axiom implies that the agent can apply it as often as he likes. As an example, suppose that an agent knows what day of the week is today, and that he also knows which day of the week it is on any given day, if he would know this about the previous day. This would imply that the agent knows which day of the week it is on 25 of August 6034! For a weaker notion like belief such criticisms are even more compelling. It is argued that humans for instance might well believe $\varphi$ in 'one frame of mind' (e.g., 'I pursuit an academic career') and something that is incompatible with it, in another ('I aim to become rich'). Some formal manifestations of logical omniscience are the axiom $\mathbf{K}$, the validity $\square(\varphi \wedge \psi) \leftrightarrow(\square \varphi \wedge \square \psi)$, the inference rule Nec and, some argue, the derived rule Eq: from $\varphi \leftrightarrow \psi$, infer $\square \varphi \leftrightarrow \square \psi$.

The idea that it should be possible to believe $\varphi$ in one frame of mind and $\neg \varphi$ in another is one of the motivating requirements that lead to neighbourhood semantics. Here, rather than states that are considered possible by the agent, we have sets of states: each such set represents a possible frame of mind the agent can be in.

Definition 4. A neighbourhood model $\mathrm{M}=\langle S, N, V\rangle$ where $S$ is a set of states and $N: \mathrm{Op} \rightarrow W \rightarrow 2^{2^{S}}$ assigns a neighbourhood $N_{\square}(s) \subseteq 2^{S}$ to every state $s$, for every operator $\square \in \mathrm{Op}$. As before, $V(p) \subseteq S$ is the valuation function of the model. The pair $\mathrm{F}=\langle S, N\rangle$ is a neighbourhood frame. Given a model $\mathrm{M}$, defining $\llbracket \varphi \rrbracket_{\mathrm{M}}$ (or simply $\llbracket \varphi \rrbracket$ if M is clear) to be $\llbracket \varphi \rrbracket=\{s \in S \mid \mathrm{M}, s \models \varphi\}$, the relevant truth condition for modal operators is

$$
\mathrm{M}, s \models \square \varphi \text { iff for some } T \in N_{\square}(s), T=\llbracket \varphi \rrbracket
$$

In terms of knowledge: $\varphi$ is known at $s$ if the denotation of $\varphi$ in $\mathrm{M}$ is one of the neighbourhoods of $s$. Neighbourhood models are more general than relational Kripke models: given $M=\langle S, R, V\rangle$ one can define $\mathrm{M}=\langle S, N, V\rangle$ by

$$
N_{\square}(s)=\{U \subseteq S: R(s) \subseteq U\} .
$$

Then, for any $s \in S$, the models $M, s$ and M,s satisfy the same formulas. The other direction does not hold: indeed, under neighbourhood semantics, the property $(\square \varphi \wedge \square \psi) \rightarrow \square(\varphi \wedge \psi)$ is not valid. If a neighbourhood model $\mathrm{M}=\langle S, N, V\rangle$ is augmented, there does exist an equivalent relational model for it, where $\mathrm{M}$ is augmented if for all $s,(1) \cap_{T \in N_{\square}(s)} T \in N(s)$, (2) $T_{1} \cap T_{2} \in N_{\square}(s)$ only if $T_{1}, T_{2} \in N_{\square}(s)$, and (3) If $T_{1} \in N_{\square}(s)$ and $T_{1} \subseteq T_{2}$, then $T_{2} \in N_{\square}(s)$. 
One can 'recover' epistemic properties like veridicality and introspection in neighbourhood semantics by putting further constraints on the neighbourhood function $N$. Moreover, it is possible to use this semantics for multi-agent logics: the notion of 'everybody knows' for instance is then the modal operator for the neighbourhood function $N_{E}=\cap_{a \in \mathrm{Ag}} N_{a}$. For common knowledge this can be done as well: we here follow [79]. It is not difficult to see that

$$
\mathrm{M}, s \models K_{a} K_{b} \varphi \text { iff }\left\{t \in S \mid \llbracket \varphi \rrbracket \in N_{b}(t)\right\} \in N_{a}(s)
$$

In order to manipulate such expressions, it is convenient to define an algebraic operator $\circ$ on neighbourhoods as follows. Let $T \subseteq S$.

$$
T \in N_{1} \circ N_{2}(s) \text { iff }\left\{t \in S \mid T \in N_{2}(t)\right\} \in N_{1}(s)
$$

Equation (8) then becomes: $\mathrm{M}, s \models K_{a} K_{b} \varphi$ iff $\llbracket \varphi \rrbracket \in N_{a} \circ N_{b}(s)$. In this context, it is best to interpret common knowledge as the infinite conjunction

$$
E \varphi \wedge E(\varphi \wedge E \varphi) \wedge E(\varphi \wedge E(\varphi \wedge E \varphi)), \ldots
$$

In normal modal logic (10) is equivalent to (2), but using a neighbourhood semantics it is not! Let the special neighbourhood system $\mathcal{E}$ be defined by $T \in \mathcal{E}(s)$ iff $s \in T$. We then have $N \circ \mathcal{E}=\mathcal{E} \circ N=N$ for every $N$. Keeping in mind (10) define now a sequence of neighbourhood systems as follows.

$$
N_{0}=N_{E} \text { and for any ordinal } \eta, N_{\eta}=N_{E} \circ\left(\bigcap_{\zeta<\eta} N_{k} \cap \mathcal{E}\right)
$$

We now assume that the systems $N_{a}$ in a model $\mathrm{M}$ are closed under supersets, i.e., $T \in N_{a}(s)$ and $T \supseteq T^{\prime}$ implies that $T^{\prime} \in N_{a}(s)$. This notion is sometimes also called monotony, and 'makes for smoother theory', quoting van Benthem et al. ([18]). On such models, we have

Lemma 3. [79, Lemma 5] Let $\xi$ and $\eta$ be ordinals. If $\xi<\eta$, then $N_{\eta} \subseteq N_{\xi}$.

By Lemma 3, for any $s \in S$ the sequence $N_{\eta}(s)$ is a decreasing sequence of sets. Hence, there is a smallest ordinal $o_{s}$ such that for all $\eta \geq o_{s}, N_{\eta}(s)=N_{o_{s}}(s)$. Now take $\delta=\sup \left\{o_{s} \mid s \in S\right\}$ : we have $N_{\eta}=N_{\delta}$ for all $\eta \geq \delta$. So the neighbourhood system against which common knowledge is interpreted is $N_{C}=N_{\delta}$.

This semantics is characterised by an axiomatisation given by [78], summarised in Table 4.

\begin{tabular}{|l|l|}
\hline \multicolumn{3}{|c|}{ Common Knowledge for neighbourhood models } \\
\hline $\mathbf{E} \quad E \varphi \leftrightarrow \bigwedge_{a \in \mathrm{Ag}} K_{a} \varphi$ & $\mathbf{F P} \quad C \varphi \rightarrow E(C \varphi \wedge \varphi)$ \\
\hline Ind From $\varphi \rightarrow E \varphi$, infer $E \varphi \rightarrow C \varphi$ & Mon From $\varphi \rightarrow \psi$, infer $\square \varphi \rightarrow \square \psi \quad \square \neq E$ \\
\hline
\end{tabular}

Table 4 The axioms and rules above are added to the propositional Taut and MP 
It is also possible to generalise the notion of bisimulation (to behavioural equivalence) to neighbourhood models, as well as to have a suitable notion of standard translation to a two-sorted first-order language, where the crucial clause for the translation is $\mathrm{ST}_{x}(\square \varphi)=\exists T\left(x \mathrm{~N} T \wedge \forall y\left(T \mathrm{E} y \leftrightarrow S T_{y}(\varphi)\right)\right)$, where $x \mathrm{~N} T$ iff $T \in N(x)$ and $T$ E $y$ iff $y \in T$. With such an apparatus in place, [60] has been able to prove a 'van Benthem-style' characterisation theorem for modal logic using a neighbourhood semantics. For completeness of modal logics wrt neighbourhood semantics we refer to e.g., [37] and [59]. An example of a logic that is Kripke incomplete, but is complete wrt neighbourhood frames can be found in [56].

Neighbourhood semantics are a very powerful tool for reasoning about games as well, if a neighbourhood is interpreted as a set of states a player can enforce. Van Benthem et al. use this semantics to define their concurrent game logic ([18], and Chapter 15 of this book). Interestingly, van Benthem and Pacuit ([22]) have given an interpretation reminiscent of the notion of groundedness (see Section 3.5) to that of neighbourhoods: rather than using neighbourhoods as a technical device to study weak modal logics, they 'concretely' interpret a neighbourhood as an 'evidence set' of an agent who then can reason about the evidence, beliefs and knowledge - and their dynamics - he entertains.

\subsection{Topological Semantics}

Next we will discuss topological semantics of epistemic and doxastic logic. Topological semantics is closely related to Kripke and neighbourhood semantics. As we will see below, the standard Kripke semantics of $S 4$ corresponds to special (Alexandroff) topological spaces. So topological semantics generalises the Kripke semantics of epistemic logic. On the other hand, topological models coincide with the neighbourhood models of $S 4$. Nevertheless, it is useful to think in topological terms as it gives us an elegant and, at the same time, powerful mathematical machinery to investigate non-standard models of epistemic logic. In topological models of intuitionistic logic, open sets are treated as 'observable properties'. In domain theory, Scott domains are special posets equipped with the so-called Scott topology, where points are interpreted as 'pieces of information' or 'results of a computation'. Modal (epistemic) logic also provides a useful formalism to reason about (topological) spaces connecting it to the area of spatial logic.

Topological semantics also brings concrete benefits to the semantics of epistemic logic as observed by van Benthem and Sarenac [23]. (1) Topological products provide a way of merging the knowledge of two agents with no new information arising. (2) More importantly, they address Barwise's criticism of the Kripke semantics as the two ways of computing common knowledge, discussed in previous sections, no longer coincide. (3) Topological products also address Barwise's other criticism of Kripke semantics about modelling shared epistemic situation. (4) Finally, in some important cases (i.e., for distributed knowledge) topological interpretations of epistemic notions nicely complement the relational interpretations. 


\subsubsection{Topological spaces: connection with Kripke and neighbourhood frames}

A topological space is a pair $(X, \tau)$, where $X$ is a non-empty set and $\tau \subseteq \mathcal{P}(X)$ contains $X$ and $\emptyset$ and is closed under finite intersections and arbitrary unions. Elements of $\tau$ are called open sets. Complements of open sets are called closed sets. An open set containing $x \in X$ is called an open neighbourhood of $x$. The interior of a set $A \subseteq X$ is the largest open set contained in $A$ and is denoted by $\operatorname{Int}(A)$. The closure of $A$ is the least closed set containing $A$ and is denoted by $\bar{A}$. In other words, $\operatorname{Int}(A)=\bigcup\{U \in \tau: U \subseteq A\}$ and $\bar{A}=\bigcap\{F: X \backslash F \in \tau, A \subseteq F\}$. It is easy to check that $\bar{A}=X \backslash \operatorname{Int}(X \backslash A)$.

A topological space $(X, \tau)$ is called an Alexandroff space if $\tau$ is closed under infinite intersections. It is easy to see that a topological space is Alexandroff iff every point has a least open neighbourhood (the intersection of all its open neighbourhoods). It is also well known that Alexandroff spaces correspond to reflexive and transitive Kripke frames. Indeed, given an Alexandroff space $(X, \tau)$ one can define a reflexive and transitive binary relation $R_{\tau}$ on $X$ by putting $x R_{\tau} y$ iff $x \in \overline{\{y\}}$ (that is, every open set that contains $x$ also contains $y$ ). Conversely, suppose $X$ is a set with a reflexive and transitive relation $R$. We say that $U \subseteq X$ is an upset if for each $x, y \in X$, $x R y$ and $x \in U$ imply $y \in U$. We define $\tau_{R}$ as the set of all upsets of $(X, R)$. Then $\left(X, \tau_{R}\right)$ is a topological space and $R(x)=\{y \in X: x R y\}$ is the least open neighbourhood containing the point $x$. Thus, $\left(X, \tau_{R}\right)$ is Alexandroff. It is easy to check that this correspondence is one-to-one. Therefore, reflexive and transitive Kripke frames can be seen as particular examples of topological spaces. This connection between reflexive and transitive orders and topologies is at the heart of the translation between the plausibility and evidence models of dynamic epistemic logic, see [22, Sec. 5] for details.

Now we will quickly review the connection between topological spaces and neighbourhood frames. Let $(X, N)$ be a neighbourhood frame satisfying the following five conditions:

1. for each $x \in X$ we have $U \in N(x)$ and $U \subseteq V$ imply $V \in N(x)$.

2. for each $x \in X$ we have $U, V \in N(x)$ implies $U \cap V \in N(x)$.

3. for each $x \in X$ we have $N(x) \neq \emptyset$.

4. for each $x \in X$ we have $U \in N(x)$ implies $x \in U$.

5. for each $x \in X$ and $U \in N(x)$ there exists $V \in N(x)$ such that $V \subseteq U$ and for each $y \in V$ we have $V \in N(y)$.

Let $(X, \tau)$ be a topological space. Then a set $A$ is called a neighbourhood of $x$ if $x \in A$ and there is an open neighbourhood $U$ of $x$ (i.e., $U \in \tau$ with $x \in U$ ) such that $U \subseteq A$. Let $N_{\tau}(x)=\{A: A$ is a neighbourhood of $x\}$. Then it is easy to check that $\left(X, N_{\tau}\right)$ is a neighbourhood frame satisfying conditions (1)-(5). Conversely, if $(X, N)$ is such that it satisfies (1)-(5) we define a topology $\tau$ on $X$ by $\tau_{N}=\{U$ : $U \in N(x)$ for each $x \in U\}$. Then $\left(X, \tau_{N}\right)$ is a topological space. Moreover, it is not difficult to check that this correspondence is one-to-one. We refer to e.g., [72, Theorem 2.6] for all the details. We would like to mention that conditions (1)-(5) are exactly those that correspond to the axioms of the modal logic $S 4$ (see, e.g., [37]). 
To be more precise the transitivity axiom ( $\square p \rightarrow \square \square p$ ) correspondence to condition $\left(5^{\prime}\right)$ below.

$5^{\prime}$ for each $x \in X$ and $U \in N(x)$ there exists $V \in N(x)$ such that for each $y \in V$ we have $U \in N(y)$.

But it is easy to show that a neighbourhood frame $(X, N)$ satisfies $(1)-\left(5^{\prime}\right)$ iff it satisfies (1)-(5). Thus, topological spaces correspond to neighbourhood frames of the modal logic $S 4$.

\subsubsection{Topological models of epistemic logic}

A triple $M=(X, \tau, v)$ is a topological model if $(X, \tau)$ is a topological space and $v$ a map from the propositional variables to $\mathcal{P}(X)$. We assume that we work with the modal language introduced in Definition 1. Truth of a formula $\varphi$ in the model $M$ at a point $x$, written as $M, x \models \varphi$, is defined inductively as follows:

$$
\begin{array}{lll}
M, x=p & \text { iff } & x \in v(p) \\
M, x=\varphi \wedge \psi & \text { iff } & M, x \models \varphi \text { and } M, x=\psi \\
M, x=\neg \varphi & \text { iff } & \text { not } M, x \models \varphi \\
M, x=\square \varphi & \text { iff } & \exists U \in \tau \text { such that } x \in U \text { and } \forall y \in U M, y \models \varphi .
\end{array}
$$

Let $\llbracket \varphi]_{v}=\{x \in X: M, x=\varphi\}$. We will skip the index if it is clear from the context. It is easy to see that the last item is equivalent to $[\square \varphi \rrbracket=\operatorname{Int}(\llbracket \varphi \rrbracket])$. Moreover, as $\diamond \varphi=\neg \square \neg \varphi$, we have that $\llbracket \diamond \varphi \rrbracket=\overline{\llbracket \varphi \rrbracket}$. A pointwise definition of the semantics of $\diamond$ is as follows:

$$
M, x=\diamond \varphi \quad \text { iff } \forall U \in \tau \text { such that } x \in U, \exists y \in U \text { with } M, y \models \varphi .
$$

Note that if $(X, \tau)$ is an Alexandroff space, then the above definition of the semantics of formulas coincides with the one defined in Section 3.1 for Kripke models. Also if we view topological models as particular examples of neighbourhood models, then the above semantics coincides with the semantics of formulas in neighbourhood models defined in Section 4.1. The notion of satisfiability and validity of formulas in topological models as well as topological soundness and completeness of logics is defined in the same way as in Section 4.1.

Let us look at an example of topological interpretations. Let $\mathbb{R}$ be the real line where a topology $\tau$ on $\mathbb{R}$ is given by open intervals and their unions. Let also $v(p)=$ $[0,1)=\{r \in \mathbb{R}: 0 \leq r<1\}$. We invite the reader to check that

- $[\square p \rrbracket=(0,1)$,

- $[[\diamond p]=[0,1]$,

- $[[\diamond p \wedge \diamond \neg p]=\{0,1\}$,

- $[p \wedge \diamond p \wedge \diamond \neg p \rrbracket=\{0\}$,

- $[[\neg p \wedge \diamond p \wedge \diamond \neg p]]=\{1\}$. 
Now we briefly discuss why topological models are of interest from the epistemic logic point of view. Topological semantics of modal logic precedes Kripke semantics and dates back to the 1930s. Already back then topological models were used to model knowledge in the context of intuitionistic logic (see e.g., [100]). Open sets can be interpreted as 'pieces of evidence', e.g., about location of a point. This reflects on the Brouwer-Heyting-Kolmogorov semantics, which informally defines intuitionistic truth as provability and specifies the intuitionistic connectives via operations on proofs. One could extend this reading to modal logic and give epistemic interpretation to $\square_{a} p$ in a topological model as: there exists a piece of evidence for agent $a$ (i.e., an open set in $a$ 's topology), which validates the proposition $p$. We point out again that in [22] neighbourhood models are used to model the evidence of agents. Thus, the topological/neighbourhood model setting does not just refine the analysis of deduction or static attitudes, but also allows for a richer repertoire of dynamic information-carrying events. As we will see below, topological models also give a (nice) way to 'naturally' merge the knowledge of different agents (see van Benthem and Sarenac [23] for more discussion on topologies as models of epistemic logic).

Finally, going a bit beyond epistemic logic, we remark that a related view of connecting topology to computer science proved to be very influential. In fact, many topological concepts provide natural interpretations to important notions of computability theory. For example, data type corresponds to a topological space, piece of data to a point, semi-decidable property (observable property, affirmable property) to an open set, computable function to a continuous map, etc. We refer to $[97,1,45,101]$ for a thorough investigation of this line of research.

\subsubsection{Topo-bisimulations}

Similarly to the relational semantics, in order to understand the expressive power of modal languages on topological models one needs to define the corresponding notion of a bisimulation. This has been done by van Benthem and Aiello in [2].

Definition 5. A topological bisimulation or simply a topo-bisimulation between two topological models $M=(X, \tau, v)$ and $M^{\prime}=\left(X^{\prime}, \tau^{\prime}, v^{\prime}\right)$ is a non-empty relation $T \subseteq$ $X \times X^{\prime}$ such that if $x T x^{\prime}$ then:

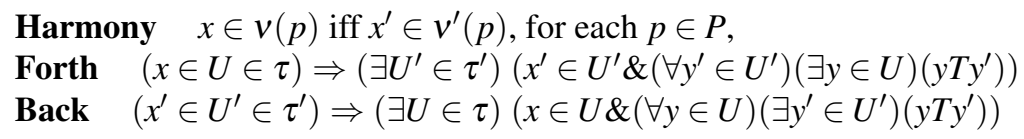

In other words $T$ is a bisimulation if $T$-image and $T$-inverse image of an open set is open. Two topological models are topo-bisimilar if there is a topo-bisimulation between them.

Let us look at some examples. First note that if two topological models are based on Alexandroff spaces, then a topo-bisimulation is the same as the standard Kripke bisimulation (Definition 3) between the corresponding reflexive and transitive Kripke models. Recall that a topology on the real line $\mathbb{R}$ is given by open 
intervals and their unions. Let $W=\left\{s, s^{-}, s^{+}\right\}$and let $\preceq$ be the reflexive closure of $\left\{\left(s, s^{-}\right),\left(s, s^{+}\right)\right\}$. Then $(W, \preceq)$ is the so-called 2-fork. Obviously the 2-fork is reflexive and transitive. So it corresponds to an Alexandroff space. It is now an easy exercise to check that the relation $T$ between $\mathbb{R}$ and $W$ defined as: $T(0, s), T\left(r, s^{-}\right)$for each $r \in \mathbb{R}$ with $r<0$ and $T\left(r, s^{+}\right)$for each $r \in \mathbb{R}$ with $r>0$, is a topo-bisimulation. In fact, there is a deeper connection between these two structures. We refer to [3] and [15] for more details on the connection of the spatial logic of $\mathbb{R}$ and the logic of the 2-fork.

Let $\mathbb{Q}$ be the set of rational numbers equipped with the topology induced from the reals. That is, open sets of $\mathbb{Q}$ are intersections of $\mathbb{R}$-open sets with $\mathbb{Q}$. Now it is easy to check that $T \subseteq \mathbb{R} \times \mathbb{Q}$ defined as: $T(z, z)$ for each $z \in \mathbb{Z}, T(r, q)$ for each $r \in \mathbb{R}$ and $q \in \mathbb{Q}$ with $z<r, q<z+1$, for each $z \in \mathbb{Z}$ is a topo-bisimulation.

In the two cases above we assumed that $v(p)=\emptyset$ for each $p$. Now consider an example where this is not the case. Let $\mathbb{R}$ and $\mathbb{R}^{\prime}$ be two isomorphic copies of the reals, with $v(p)=[0,1]$ and $v^{\prime}(p)=\left(0^{\prime}, 1^{\prime}\right)$. Then there is no topo-bisimulation between $(\mathbb{R}, v)$ and $\left(\mathbb{R}^{\prime}, v^{\prime}\right)$ relating 0 to any point $r^{\prime} \in \mathbb{R}^{\prime}$. To see this, note that by the basic case of the topo-bisimulation, $r^{\prime} \in\left(0^{\prime}, 1^{\prime}\right)$. But then, by the forth condition, for $U^{\prime}=\left(0^{\prime}, 1^{\prime}\right)$ there exists a neighbourhood $U$ of 0 such that every point in $U$ is topo-bisimilar to some point in $\left(0^{\prime}, 1^{\prime}\right)$. But this is impossible as each neighbourhood of 0 contains a point $t<0$. Then $t$ cannot be topo-bisimilar to any point in $\left(0^{\prime}, 1^{\prime}\right)$ as $t$ does not satisfy $p$. Thus, there is no topo-bisimulation between $(\mathbb{R}, v)$ and $\left(\mathbb{R}^{\prime}, v^{\prime}\right)$ relating 0 to some point in $\mathbb{R}^{\prime}$.

Similarly to bisimilar Kripke models, topo-bisimilar models satisfy the same modal formulas. That is, if $x \in X$ and $x^{\prime} \in X^{\prime}$ and $x T x^{\prime}$, then $M, x \models \varphi$ iff $M^{\prime}, x^{\prime} \models \varphi$, for each formula $\varphi$. The converse is true for finite models. The notion of bisimilarity can also be expressed using games (see e.g., [2]).

The celebrated van Benthem's characterisation theorem (Theorem 3), states that on Kripke models modal logic is the bisimulation invariant fragment of first-order logic. An analogue of this theorem for topo-bisimulations and the language $L_{t}$ (an analogue of the first-order language for topological spaces) was proved in [35].

\subsubsection{Topological Completeness}

Now we turn to deductive systems and the issues of axiomatisation and topological completeness. Note that the interior and closure operators satisfy the following wellknown Kuratowski axioms (see e.g., [41]):

$$
\begin{array}{ll}
\text { 1. } \operatorname{Int}(X)=X, & \bar{\emptyset}=\emptyset, \\
\text { 2. } \operatorname{Int}(A \cap B)=\operatorname{Int}(A) \cap \operatorname{Int}(B), & \overline{A \cup B}=\bar{A} \cup \bar{B} . \\
\text { 3. } \operatorname{Int}(A) \subseteq A & A \subseteq \bar{A} . \\
\text { 4. } \operatorname{Int}(A) \subseteq \operatorname{Int}(\operatorname{Int}(A)), & \overline{\bar{A} \subseteq \bar{A} .}
\end{array}
$$

It is easy to see that the above implies that $S 4$ is sound with respect to topological semantics. In fact, 1-4 above are the axioms of $S 4$ translated into topological 
terms. For completeness, we need to show that if $\varphi$ is $S 4$-consistent, then there exists a topological model $(X, \tau, v)$ satisfying $\varphi$. As we know (e.g., by the standard canonical Kripke model argument), if $\varphi$ is $S 4$-consistent, then $\varphi$ is satisfiable in a Kripke model with a reflexive and transitive relation. As every reflexive and transitive Kripke frame corresponds to an Alexandroff space, the completeness follows. Van Benthem and his collaborators, however, gave a different, elegant and more self-contained proof of this result by introducing a topo-canonical model (a topological analogue of a canonical Kripke model) [3], [14]. We will quickly sketch the basic idea of this construction.

The topo-canonical model of $S 4$ (in fact, instead of $S 4$ we can consider any logic $L$ over $S 4)$ is a triple $M^{\mathcal{C}}=\left(X^{\mathcal{C}}, \tau^{\mathcal{C}}, v^{\mathcal{C}}\right)$, where $X^{\mathcal{C}}$ is the set of all maximal $S 4$ consistent sets. Elements of $\tau^{\mathcal{C}}$ are unions of the sets $U_{\varphi}=\left\{\Gamma \in X^{\mathcal{C}}: \square \varphi \in \Gamma\right\}$. In other words, $\left\{U_{\varphi}: \varphi\right.$ is any formula $\}$ forms a basis for $\tau^{\mathcal{C}}$. Finally, we put $\Gamma \in v^{\mathcal{C}}(p)$ if $p \in \Gamma$. Then $\left(X^{\mathcal{C}}, \tau^{\mathcal{C}}, v^{\mathcal{C}}\right)$ is a topological model. Moreover, the Truth Lemma holds for this model. That is,

$$
M^{\mathcal{C}}, \Gamma \models \varphi \text { iff } \varphi \in \Gamma .
$$

Now if $\varphi$ is $S 4$-consistent, then by the Lindenbaum Lemma (see, e.g., [32], [36]) $\{\varphi\}$ can be extended to a maximal $S 4$-consistent set $\Gamma$. By the Truth Lemma, $M^{\mathcal{C}}, \Gamma \models \varphi$, which finishes the proof.

As mentioned above, the topo-canonical model construction can be defined for any normal modal logic $L$ extending $S 4$. In analogy with the relational case, in the topological setting too one can define the notion of canonicity. A logic $L \supseteq S 4$ is called topo-canonical if the topo-canonical model of $L$ is based on a topological space validating $L$. Topo-canonical logics have been thoroughly investigated in [30].

So $S 4$ is sound and complete with respect to all topological spaces. However, next question is whether one can find 'good' topological spaces for which $S 4$ is sound and complete. The classical result of McKinsey and Tarski [85] states that $S 4$ is sound and complete with respect to any dense-in-itself metrizable separable space. This includes the real line $\mathbb{R}$, and in general any Euclidean space $\mathbb{R}^{n}$, the Cantor space $\mathbf{C}$, the space of rational numbers $\mathbb{Q}$, etc. There is a number of different proofs of completeness of $S 4$ with respect to these structures, see e.g., [87], [3], [88], [28], [68] and [14] for an overview. Here we only give a sketch of the basic idea underlying most of these proofs. Strong completeness of $S 4$ with respect to any dense-in-itself metric separable space was recently shown in [76] and [77]. A full axiomatization of the space of rational numbers $\mathbb{Q}$ in the language with topological and temporal modalities $F$ and $P$ was given in [95]. Recently, [69] gave a full axiomatization of the real line $\mathbb{R}$ in the same language.

As $S 4$ is sound with respect to all topological spaces, it is obviously sound with respect to each of the topological spaces mentioned above. For completeness, assume that $\varphi$ is $S 4$-consistent. Then as $S 4$ has the finite model property (see e.g., $[32,36])$ there exists a finite rooted, model $M=(W, R, V)$, with a root $r$, such that $R$ is reflexive and transitive and $M, r \models \varphi$. Sometimes it is useful to exploit here the completeness of $S 4$ with respect to other structures, say an infinite binary tree, etc. As $R$ is reflexive and transitive, $(W, R)$ could be viewed as an Alexandroff space and, 
thus, $M$ is a topological model. Now let $(X, \tau)$ be the topological space for which we want to prove the completeness of $S 4$ (e.g., $\mathbb{R}, \mathbb{Q}, \mathbf{C}$, etc.). If we manage to define a valuation $v$ on $X$ so that $M$ and $(X, \tau, v)$ are topo-bisimilar, then as topo-bisimilar points satisfy the same formulas, $(X, \tau, v)$ will satisfy $\varphi$. In order to show that such a valuation and bisimulation exist, it is sufficient to prove that there exists a continuous and open map $f: X \rightarrow W$. Recall that $f$ is continuous if the inverse $f$-image of an open set is open, and $f$ is open if the direct $f$-image of an open set is open. Suppose such a map exists. Then we define $v(p)=f^{-1}(V(p))$. Moreover, the graph of this map will be a topo-bisimulation. Thus, the proof of completeness is reduced to defining a continuous and open map. This is not an easy task and there are many different constructions for different topological spaces. We refer to [14], and the references therein, for the details on this and on the topological completeness results obtained via this method.

So far we saw only one (epistemic) logical system associated with topological semantics - the modal logic $S 4$. In the next section we will discuss few different ways of obtaining (epistemic) topological logics beyond $S 4$. We want to reiterate that the ideas and insights of van Benthem were instrumental in advancing these research directions.

First we briefly discuss topological models with restricted valuations. When evaluating formulas in, for example, the real line $\mathbb{R}$, instead of the whole powerset, one could consider evaluating formulas as intervals and their finite (or countable) unions. In the real plane one could take (unions) of convex sets, polygons, or rectangles. Such evaluations, and corresponding logics have been studied in [103], [3] and [15], see also [14]. In particular, [15] shows that such restricted valuation can capture the difference between dimensions of Euclidean spaces. This cannot be done with standard interpretations as the logic of any Eucledean space is $S 4$. We refer to [73] for a thorough study of the computational aspects of the logics arising from Eucledean spaces.

\subsubsection{Topological products}

Taking products is a natural way of combining two Kripke complete modal logics [51]. The problem with this is that the resulting logic might become much more complex than the original ones. For example, the logic $S 4 \times S 4$ is undecidable [53], whereas $S 4$ is decidable. Moreover, when taking products of Kripke frames, the resulting frame always validates the extra axioms of commutativity $\left(\square_{1} \square_{2} p \leftrightarrow \square_{2} \square_{1} p\right)$ and Church-Rosser $\left(\diamond_{1} \square_{2} p \rightarrow \square_{2} \diamond_{1} p\right)$. In [16] van Benthem and his collaborators defined topological products of topological spaces. We briefly recall this construction.

Let $(X, \tau)$ and $\left(X^{\prime}, \tau^{\prime}\right)$ be topological spaces. Suppose $A \subseteq X \times X^{\prime}$. We say that $A$ is horizontally open (in short, H-open) if for any $\left(x, x^{\prime}\right) \in A$, there exists $U \in \tau$ such that $x \in U$ and $U \times\left\{x^{\prime}\right\} \subseteq A$. Vertically open sets (in short, V-open sets) are defined similarly. We let $\tau_{1}$ and $\tau_{2}$ denote the collection of all horizontally and vertically open subsets of $X \times X^{\prime}$, respectively. It is easy to see that $\tau_{1}$ and $\tau_{2}$ are 
topologies. Modal operators $\square_{1}$ and $\square_{2}$ in a product model $M=\left(X \times X^{\prime}, \tau_{1}, \tau_{2}, v\right)$ are interpreted as follows.

$$
\begin{aligned}
& M,\left(x, x^{\prime}\right) \models \square_{1} \varphi \quad \text { iff } \exists U \in \tau_{1} \text { such that } x \in U \text { and } \forall y \in U, M,\left(y, x^{\prime}\right) \models \varphi . \\
& M,\left(x, x^{\prime}\right) \models \square_{2} \varphi \quad \text { iff } \exists U \in \tau_{2} \text { such that } x^{\prime} \in U \text { and } \forall z \in U, M,(x, z) \models \varphi .
\end{aligned}
$$

Consider as an example $\left(\mathbb{R} \times \mathbb{R}, \tau_{1}, \tau_{2}\right)$, and let $v(p)=[0,1) \times\{0\}$. Then it is easy to see that

- $\left[\left[\square_{1} p\right]=(0,1) \times\{0\}\right.$,

- $\left[\left[\diamond_{1} p\right]\right]=[0,1] \times\{0\}$,

- $\left[\square_{2} p\right]=\emptyset$,

- $\left[\left[\diamond_{2} p\right]\right]=[0,1) \times\{0\}$.

This operation can be extended to a notion of a product of two topologically complete modal logics. Given topologically complete uni-modal $\operatorname{logics} L$ and $L^{\prime}$, their topological product is the bi-modal logic of the product frames $X \times X^{\prime}$, where $X$ is a topological frame for $L$ and $X^{\prime}$ for $L^{\prime}$, respectively.

Surprisingly enough, topological products turned out to be very well behaved. [16] shows that the topological product of $S 4$ with itself is the same as the logic $S 4 \otimes S 4, S 4$ fusion $S 4$, which is decidable. The fusion is just the smallest bi-modal logic that contains $S 4$-axioms for both modalities. Thus, no extra axiom is valid on product topological spaces. The Church-Rosser and commutativity axioms can be refuted on the product space $\mathbb{R} \times \mathbb{R}$. Moreover, the logic $S 4 \otimes S 4$ is sound and complete with respect to the product space $\mathbb{Q} \times \mathbb{Q}$. It is still an open question to find an axiomatisation of the logic of $\mathbb{R} \times \mathbb{R}[75]$.

One could view topological products of epistemic logics as a new and interesting way of merging the knowledge of two agents [23]. The fact that unlike relational products, no new axiom is valid in the topological case, shows that when we 'topologically merge' (via taking topological products of uni-modal epistemic logics) the knowledge of two agents, no new information arises. Topological products have other nice properties. Recall that in relational semantics the distributed knowledge of agents (which describes what a group would know if its members decided to merge their information) is expressed by taking the $D_{A}$ operator - the Box operator of the intersection of the relations. In topological products one expresses distributed knowledge by taking the interior of the join of the topologies. By the join of two topologies we mean the least topology that contains both topologies. We note that in the topological case the join is not always very interesting e.g., for $\mathbb{Q} \times \mathbb{Q}$, the join of horizontal and vertical topologies is just the discrete topology (all sets are open). We refer to [23] for all the details on this.

However, there is not always an analogy between relational and topological semantics of epistemic logic. In fact, (in some way) topological models provide a richer landscape for interpreting epistemic logic, than the relational semantics. In his well-known paper [6] Barwise underlined that a proper analysis of common knowledge must distinguish the following three approaches:

1. countably infinite iteration of individual knowledge modalities, 
2. the fixed point view of common knowledge as 'equilibrium',

3. agents having a shared epistemic situation.

The relational semantics of epistemic logic cannot properly distinguish these three approaches (see Section 3.1), whereas topological semantics (topological products) suits this purpose perfectly well. Recall that in the Kripke semantics the approximation of the common knowledge operator stabilises in $\kappa \leq \omega$ steps. It was noted by van Benthem and Sarenac [23] that this is no longer the case in topological semantics, thus addressing Barwise's criticism.

In topological models common knowledge as equilibrium is expressed by taking the intersection of topologies. We will quickly sketch this argument. Let $\tau_{1}$ and $\tau_{2}$ be two topologies. Here we do not need to assume that these topologies are the vertical and horizontal topologies of a product space. Recall from the previous section that $C \varphi=v x\left(\varphi \wedge \square_{1} x \wedge \square_{2} x\right)$. Let $\psi=\varphi \wedge \square_{1} x \wedge \square_{2} x$. Then $C \varphi=v x \psi$. As it was also discussed in the previous section, $\left[[C \varphi \rrbracket]_{V}=\llbracket[v x \psi]_{V}=\bigcup\{U \in \mathcal{P}(W): U \subseteq \llbracket \psi]_{V_{x}^{U}}\right\}$. Now observe that for each $i=1,2$ we have $\left[\left[\square_{i} x\right]_{V_{U}}=\operatorname{Int}_{\tau_{i}}(U)\right.$. Hence, for $i=1,2$ we have $U \subseteq\left[\square \square_{i} x \rrbracket\right]_{V_{x}^{U}}$ iff $U$ is $\tau_{i^{-}}$-open. Thus, $\left.U \subseteq \llbracket \psi\right]_{V_{x}^{U}}$ iff $U \subseteq[\varphi]_{V}$ and $U$ is $\tau_{1}$ and $\tau_{2}$-open. Therefore, $\left.[\llbracket C \varphi]_{V}=\bigcup\{U \subseteq \llbracket \varphi]_{V}: U \in \tau_{1} \cap \tau_{2}\right\}=\operatorname{Int}_{\tau_{1} \cap \tau_{2}}(\llbracket \varphi \rrbracket)$. So topologically the common knowledge corresponds to the interior of the intersection of the topologies.

It is proved in [23] that the (countably) infinite iterations of the individual knowledge modalities may not be (horizontally or vertically) open. Hence, computing $C \varphi$ as fixed equilibrium and as countable iterations of $\varphi \wedge E \top$ in topological models diverge. This fact captures the difference between (1) and (2) above. A similar observation was made in [79, Proposition 4] for neighbourhood frames.

In a topological setting one can also analyse a 'shared situation' when there is a new group concept $\tau$ that only accepts very strong collective evidence for any proposition. This corresponds to adding the standard product topology on top of the horizontal and vertical topologies. Thus, formally speaking, we have three operators, the horizontal and vertical $\square_{1}, \square_{2}$ and also $\square$ of the standard product topology. This again addresses Barwise's critical comments on common knowledge in the topological setting by showing that (3) can also differ from (1) and (2). It was proved in [16] that the logic of such spaces is the ternary modal logic obtained by adding the axiom $\square p \rightarrow\left(\square_{1} p \wedge \square_{2} p\right)$ to the three dimensional fusion logic $S 4 \otimes S 4 \otimes S 4$.

Next we give an example that illustrates how to compute topologically common and shared knowledge. This example also shows that these two notions differ. Consider the topological product $\mathbb{R} \times \mathbb{R}$ with horizontal and vertical topologies. Let $\square$ be interpreted as the interior of the standard product topology. We let $v(p)$ be the 'cross' or the 'pair of orthogonal bow ties' depicted in Figure 4.2.5 (we leave it up to the readers imagination to derive an appropriate name for this valuation). We assume that $v(p)$ has no boundaries, but the point $(0,0)$ belongs to it. A very similar example can be found in [14], [16] and [23]. Obviously one could give a formal mathematical description of $v(p)$, but we prefer to stick with a picture that provides sufficient intuition. Then it is easy to check that

- $\left[\left[\square_{1} p\right]=\left[\left[\square_{2} p\right]\right]=\llbracket[C p]=v(p)\right.$, 
- $[[\square p]=v(p) \backslash\{(0,0)\}$.

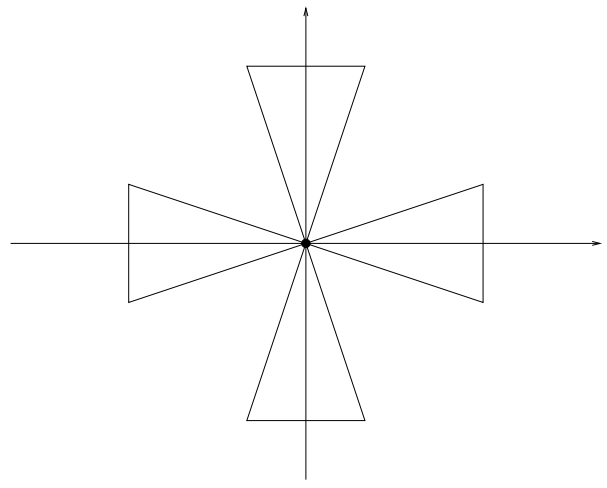

Fig. 4 The cross-valuation on the product space

Finally, we note that another epistemic logic $S 5$ also admits topological semantics, but much more exotic one than $S 4$. We say that a topological space is a clopen space if every closed subset of it is also open. Examples of such spaces are discrete spaces (every subset is open), and also the spaces with the trivial topology (only the empty set and the whole space are open). We recall that $S 5$ can be obtained from $S 4$ by adding the axiom $p \rightarrow \square \diamond p$ or alternatively by adding the axiom $\diamond p \rightarrow \square \diamond p$. Translating this into topological terms, a topological space $(X, \tau)$ validates $S 5$ iff $\bar{A} \subseteq \operatorname{Int}(\bar{A})$ for each $A \subseteq X$. This is equivalent to the fact that every closed subset of $X$ is open. This means that $S 5$ is sound with respect to clopen spaces. For the topological completeness of $S 5$ one can apply Kripke completeness of $S 5$ and the argument that was used in the topological completeness of $S 4$, see [14] for the details.

For the topological completeness of other extensions of $S 4$ such as the logic $S 4 . G r z$ we refer to [43] and [29]. Questions on modal definability of (classes) of topological spaces were studied in [52]. Topological completeness of $S 4$ with the universal modality with respect to connected spaces was proved in [96] and further generalised in [28]. Measure-theoretic semantics of this logic can be found in [47].

Another approach (similar in essence, but different in technicalities) to topological interpretations of epistemic logic goes via the so-called subset spaces [90]. This interpretation uses a bimodal language. This led to introducing topologic - the axiomatic system (in this extended language) sound and complete wrt all topological spaces $[54,55]$. Completeness of topologic via canonical model construction was proved in [38]. We will not discuss this approach here, but will instead refer to an overview article [91] for all the details and references. Further generalisations and stronger completeness results for topologic and multi-agent epistemic logics of subset spaces have been obtained by Heinemann in [62, 63, 65, 64]. However, Heinemann achieves this by adding more than 'just' several knowledge operators 
$K_{i}$ to subset space logic: they are definable from other operators that are added, and they do not have the $S 5$ properties of knowledge. Only recently, Wang ad Ågotnes ([102]) gave a complete axiomatisation for multi-agent epistemic subset space logic as a generalisation of the single agent case.

\subsubsection{Topological models of doxastic logic}

In this section we discuss a different topological semantics of modal logic via the derived set operator. As we will see below this semantics admits doxastic interpretations, nicely complementing the epistemic semantics of the closure and interior operators discussed in the previous section. We will again concentrate on the issues of expressivity (bisimulations) and topo-completeness for this semantics.

Let $(X, \tau)$ be a topological space. We recall that a point $x$ is called a limit point (limit points are also called accumulation points) of a set $A \subseteq X$ if for each open neighbourhood $U$ of $x$ we have $(U \backslash\{x\}) \cap A \neq \emptyset$. A point $x \in A$ is called an isolated point of $A$ if $x \notin d(A)$. Let $d(A)$ denote all limit points of $A$. This set is called the derived set and $d$ is called the derived set operator. For each $A \subseteq X$ we let $t(A)=X \backslash d(X \backslash A)$. We call $t$ the co-derived set operator. Also recall that there is a close connection between the derived set operator and the closure operator. In particular, for each $A \subseteq X$ we have $\bar{A}=A \cup d(A)$. Thus, the derived set operator is more expressive than the closure operator, see [25] for a discussion on this. Unlike the closure operator there may exist elements of $A$ that are not its limit points. In other words, in general $A \nsubseteq d(A)$. To see this, consider the real line $\mathbb{R}$ and let $A=[0,1] \cup\{2\}$. Then $2 \notin d(A)$, but $2 \in A$.

Let $(X, \tau)$ be an Alexandroff space. That is, $\tau$ is the set of all upsets for some reflexive and transitive relation $R$. By spelling out the definition of the derived set, we observe that $x \in d(A)$ for some $A \subseteq X$ iff there is $y \in A$ such that $x \neq y$ and $x R y$. So $d(A)=\{x \in X: \exists y \in A$, such that $x R y \& x \neq y\}$ (see [44] for more details on the derived set operator in Alexandroff spaces).

Let $M=(X, \tau, v)$ be a topological model. We now define a new semantics for $\square$ and $\diamond$ using the derived set operator. All the Boolean cases are the same as before, the only difference is in the way the modal operators are interpreted.

$$
\begin{array}{ll}
M, x=\square \varphi & \text { iff } \exists U \in \tau \text { such that } x \in U \text { and } \forall y \neq x \text { with } y \in U, M, y=\varphi . \\
M, x=\diamond \varphi & \text { iff } \forall U \in \tau \text { such that } x \in U, \exists y \neq x \text { with } y \in U \text { and } M, y=\varphi .
\end{array}
$$

We again assume that $\llbracket \varphi \varphi]_{v}=\{x \in X: M, x=\varphi\}$, and we skip the index if it is clear from the context. It is easy to see that $[\square \varphi]=t(\llbracket \varphi])$ and $[\llbracket \diamond \varphi]=d(\llbracket \varphi \rrbracket)$.

In this context too the main questions to ask are what are bisimulations for topological models with this new interpretation and what kind of completeness results one can obtain. We first briefly address the bisimulation issue and then move to completeness and doxastic interpretations. In fact, the notion of a $d$-bisimulation of topological models is the same as a topo-bisimulation with the only difference that in Definition 5 in the forth and back conditions we add $y^{\prime} \neq x^{\prime}$ and $y \neq x$. We call 
these bisimulations $d$-bisimulations. The notion of $d$-bisimilarity is defined similarly to topo-bisimilarity. Then one can prove that $d$-bisimilar points satisfy the same modal formulas. [25] defines $d$-morphisms and shows that $d$-morphisms are functional $d$-bisimulations. ( $d$-morphism is a continuous and open map such that the inverse image of each point is a discrete space in the induced topology. This definition turned out to be very useful as checking whether a map between two topological spaces is a $d$-morphism is relatively easy.)

Fixed point operators have an interesting role to play in topological semantics of derived set operator as well. For example, consider a simple formula $\mu x \square x$. We note again that we interpret fixed point formulas in topological structures in the same way as in Kripke structures (see Section 3.1). Then $\mu x \square x$ is valid in a topological space $X$ iff $X$ is scattered. To see this, recall that $X$ is scattered iff every non-empty subsets $U$ of $X$ has an isolated point. That is, for $U \subseteq X$,

$$
U \neq \emptyset \Rightarrow U \backslash d(U) \neq \emptyset .
$$

It was noted in Esakia [43] that (a logical formulation) of (12) is equivalent (over topological spaces and also over transitive Kripke frames) to the Gödel-Löb axiom ( $\square(\square p \rightarrow p) \rightarrow \square p$ ). Now it is easy to see that (12) is equivalent to the following, for $U \subseteq X$,

$$
U \neq X \Rightarrow t(U) \nsubseteq U
$$

Thus, the only subset $U$ of $X$ such that $t(U) \subseteq U$ is the whole space $X$. Therefore,

$$
X=\bigcap\{U \subseteq X: t(U) \subseteq U\}=\bigcap\left\{U \subseteq X:[[\square x]]_{V_{x}^{U}} \subseteq U\right\}=[[\mu x \square x]] .
$$

So $X$ is scattered iff $\mu x \square x$ is valid in $X$. As $\mu x \square x$ has no free variables, validity and satisfiability for this formula are equivalent.

The fact that scatteredness of a space can be captured by fixed point formulas is not very surprising as scatteredness is a topological analogue of dual wellfoundedness [43] and it was shown in [11] (see also [17]) that $\mu x \square x$ together with the transitivity axiom expresses dual well-foundedness of a Kripke structure. A similar observation in algebraic terms (for the so-called diagonalisable algebras) has been made already in [57].

Now we turn to the issue of soundness and completeness. First recall (see e..g., [41]) that the derived and co-derived set operator satisfy the following axioms.

$$
\begin{array}{ll}
\text { 1. } t(X)=X, & d(\emptyset)=\emptyset, \\
\text { 2. } t(S \cap T)=t(S) \cap t(T), & d(S \cup T)=d(S) \cup d(T) . \\
\text { 3. } A \cap t(A) \subseteq t(t(A)) & d(d(A)) \subseteq A \cup d(A) .
\end{array}
$$

Recall that $w K 4$ is a modal logic obtained form the basic modal logic $K$ by adding to it the following weak transitivity axiom $(p \wedge \square p) \rightarrow \square \square p$. The logic $w K 4$ is sound and complete with respect to Kripke frames with weakly transitive relations, where the relation is weakly transitive if $x R y, y R z$ and $x \neq z$ imply $x R z$ [44]. 
The three properties of derived and co-derived set operators listed above imply that $w K 4$ is sound with respect to topological semantics. In fact, Esakia [44] proved that $w K 4$ is also complete with respect to all topological spaces. A topological space $(X, \tau)$ is said to satisfy the $T_{D}$-separation axiom (is a $T_{D}$-space, for short) if every point of $X$ is the intersection of a closed and an open set. In fact, this condition is equivalent to $d(d(A)) \subseteq d(A)$, for each $A \subseteq X$ [41]. This implies that $K 4$ is sound with respect to $T_{D^{-}}$-spaces. [44] showed that $K 4$ is also complete with respect to $T_{D^{-}}$ spaces. For topological $d$-semantics of the provability logic $G L$, polymodal provability logic GLP, the logics of the rationals, real line and Euclidean spaces, as well as the logics of all $T_{0}$-spaces, Stone and spectral spaces and many more, we refer to [43, 44, 42, 94, 83, 82, 84, 68, 7, 25, 26, 27, 14].

We close this section by reviewing topological completeness of the doxastic modal logic $K D 45$, see [98] and [91]. The fact, mentioned above, that in general $A \nsubseteq \subseteq d(A)$ yields that the reflexivity axiom $p \rightarrow \diamond p$ (equivalently $\square p \rightarrow p$ ) is not sound with respect to this semantics, which makes this semantics suitable for doxastic logic. Recall that Kripke frames of KD45 are serial, transitive and Euclidean. As we saw above, the topological reading of the transitivity axiom gives us $T_{D^{-}}$ spaces. It is well known that the seriality axiom $\square p \rightarrow \diamond p$ is equivalent to $\diamond T$. Translating this into the topological terms we obtain the condition $d(X)=X$. This means that every point of $X$ is a limit point. Such spaces are called dense-in-itself. Finally, the topological reading of the Euclidean axiom $\diamond p \rightarrow \square \diamond p$ results in the condition $d(A) \subseteq t(d(A))$. It is easy to see that a set $A$ is closed iff $d(A) \subseteq A$. Dualising this, we obtain that a set $A$ is open iff $A \subseteq t(A)$. Thus, $d(A) \subseteq t(d(A))$ is equivalent to $d(A)$ being open.

The above leads to the following definition. A topological space $(X, \tau)$ is called a $D S O$-space ${ }^{2}$ if it is dense-in-itself $T_{D}$-space such that $d(A)$ is an open set for each $A \subseteq X$. The discussion above shows that $K D 45$ is sound with respect to DSO-spaces. Next we give an example of a DSO-space. Let $(\mathbb{N}, \tau)$ be the set $\mathbb{N}$ of natural numbers equipped with the topology $\tau=\{\emptyset$, all cofinite sets $\}$. Then it is not hard to check that for each $A \subseteq \mathbb{N}$ we have

$$
d(A)= \begin{cases}\emptyset, & \text { if } A \text { is finite } \\ \mathbb{N}, & \text { if } A \text { is infinite }\end{cases}
$$

This implies that $(\mathbb{N}, \tau)$ is a DSO-space.

Now we turn to the issue of completeness of $K D 45$ with respect to DSO-spaces. First note that with every weakly transitive frame $(X, R)$ we can associate a topology $\tau_{R}$ of all $R$-upsets. Observe that this topology will be the same as the topology of all upsets of the reflexive closure of $R$. Indeed, $A \subseteq X$ is an upset iff it is an upset for the reflexive closure of $R$.

Let $(X, R)$ be a $K D 45$-frame (that is, serial, Euclidean, transitive). Note that $\left(X, \tau_{R}\right)$ defined above, in general, is not a DSO-space. In fact, it is a DSO-space iff there are no distinct points $x, y \in X$ such that $x R y$. To see this, note that if such points exist, then $d(\{y\})$ is not an upset: we have $x \in d(\{y\})$, and $x R y$, but $y \notin d(\{y\})$ (use

\footnotetext{
${ }^{2}$ DSO stands for Derived Sets are Open.
} 
the definition of $d$ on Alexandroff spaces in the beginning of this section). So $\{y\}$ is such that $d(\{y\})$ is not open. Thus, $(X, R)$ does not correspond to a DSO-space. The converse direction is similar. Therefore, we cannot use directly the Kripke completeness of $K D 45$ for deriving its topological completeness, as we did for $S 4$. Nevertheless, one could still use Kripke completeness of $K D 45$ to obtain topological completeness. We sketch the proof. All the details can be found in [98] and [91].

Assume that $\varphi$ is $K D 45$-consistent formula. Then, by Kripke completeness of $K D 45$, there exists a Kripke model $(W, R, v)$, where $R$ is serial, transitive and Euclidean relation such that $(W, R, v)$ satisfies $\varphi$. Now let us take a product of this frame (seen as the Alexandroff space) with the DSO-space $(\mathbb{N}, \tau)$ discussed above. Then one can show that $\mathbb{N} \times W$ is a DSO-space (with the standard product topology). Moreover, the second projection is a $d$-morphism, and hence its graph is a $d$ bisimulation. Here we use the notion of a $d$-morphism between topological spaces and Kripke frames [25]. A map between a topological space and Kripke frame is called a $d$-morphism if it is continuous and open, the inverse image of an irreflexive point is a discrete subspace and the inverse image of a reflexive point is a dense-initself subspace. So $\mathbb{N} \times W$ satisfies $\varphi$, which proves the completeness of $K D 45$ for DSO-spaces.

In fact, one can strengthen this result and give an alternative proof of completeness avoiding products. [31] gives a characterisation of rooted $K D 45$ frames. Using this characterisation it is easy to see that there exists a $d$-morphism from $(\mathbb{N}, \tau)$ to any rooted $K D 45$-frame. This implies that if a formula $\varphi$ is $K D 45$-consistent, then it is satisfied in a DSO-space $(\mathbb{N}, \tau)$. Thus, $K D 45$ is sound and complete with respect to not only all DSO-spaces, but also with respect to just $(\mathbb{N}, \tau)$.

We hope that all these results illustrate that topological spaces provide interesting and insightful semantics for both epistemic and doxastic modal logic.

\section{Conclusion}

In this chapter, we have focussed on modal logics for knowledge and belief, especially their semantics. Starting with epistemic Kripke structures, we showed how Johan's results on correspondence theory often makes it possible to build an epistemic logic to which one can add a number of appealing axioms. Correspondence theory then makes it possible to quickly come up with classes of Kripke models wrt which those logics are sound and complete. Also, using a Kripke model for knowledge, it is conceptually simple to add relations to such a model that model time, or some other kind of dynamics. Johan, with his collaborators, has contributed to this field by showing how several of such dynamic epistemic logics are related. Their epistemic temporal frames provide a broad class of structures to which one can related interpreted systems, and logics for updates and revision.

Having a class of structures at hand, natural questions are when two structures are different, and what can be expressed in that class. Johan's characterisation theorem 
gives an answer for the case of normal modal logics, we have shown in this chapter how this theorem has been adapted or generalised to other classes of structures.

An important class of structures for epistemic logic is obtained by moving to a so-called neighbourhood semantics, or the closely related semantics based on a topology. Those semantics give an alternative and independent view on epistemic logic. The latter for instance can discriminate three aspects of common knowledge, which seem to be intertwined under the Kripke semantics.

Johan's contribution to logics of knowledge and belief is to be found in the technical results he has provided in the field of modal logic in general and that of epistemic logic in particular, but equally important are the themes he has consistently pursued: knowledge and ignorance are mostly interesting in a multi-agent setting, they only come to live in a dynamic context, and, while there is a multitude of schools studying epistemic logic, a close analysis tells us that they have more in common than even those schools themselves often tend to think!

Acknowledgement The first author would like to thank Guram Bezhanishvili, David Gabelaia, Ian Hodkinson, Clemens Kupke and Levan Uridia for many interesting discussions and useful suggestions. Thanks also go to Aybuke Ozgür for pointing out a few small errors. The first author would also like to acknowledge the support of the Netherlands Organization for Scientific Research grant 639.032.918 and the Rustaveli Science Foundation of Georgia grant FR/489/5-105/11.

\section{References}

1. S. Abramsky. Domain theory in logical form. Ann. Pure Appl. Logic, 51(1-2):1-77, 1991.

2. M. Aiello and J. van Benthem. A modal walk through space. J. Appl. Non-Classical Logics, 12(3-4):319-363, 2002. Spatial logics.

3. M. Aiello, J. van Benthem, and G. Bezhanishvili. Reasoning about space: the modal way. $J$. Logic Comput., 13(6):889-920, 2003.

4. R. J. Aumann. Agreeing to disagree. Annals of Statistics, 4(6):1236-1239, 1976.

5. R. J. Aumann. Interactive epistemology I: Knowledge. International Journal of Game Theory, 28:263-300, 1999.

6. J. Barwise. Three views of common knowledge. In Proceedings of the Second Conference on Theoretical Aspects of Reasoning about Knowledge (Pacific Grove, CA, 1988), pages 365-379, Los Altos, CA, 1988. Morgan Kaufmann.

7. L. Beklemishev and D. Gabelaia. Topological completeness of the provability logic GLP. arXiv: 1106.5693v1, 2011.

8. J. van Benthem. Modal Correspondence Theory. PhD thesis, University of Amsterdam, 1976.

9. J. van Benthem. Logics for information update. In Proceedings of the 8th conference on Theoretical aspects of rationality and knowledge, TARK '01, pages 51-67, San Francisco, CA, USA, 2001. Morgan Kaufmann Publishers Inc.

10. J. van Benthem. One is a lonely number: on the logic of communication. Technical report, ILLC, University of Amsterdam, 2002. Report PP-2002-27 (material presented at the Logic Colloquium 2002).

11. J. van Benthem. Modal frame correspondences and fixed-points. Studia Logica, 83(1/3):pp. 133-155, 2006. 
12. J. van Benthem. Open problems in logical dynamics. In Dov M. Gabbay, Sergei S. Goncharov, and Michael Zakharyaschev, editors, Mathematical Problems from Applied Logic I, volume 4 of International Mathematical Series, pages 137-192. Springer New York, 2006. 10.1007/0-387-31072-X_3.

13. J. van Benthem. Logical Dynamics of Information and Interaction. Cambridge Book University Press, 2011.

14. J. van Benthem and G. Bezhanishvili. Modal logics of space. In Handbook of spatial logics, pages 217-298. Springer, Dordrecht, 2007.

15. J. van Benthem, G. Bezhanishvili, and M. Gehrke. Euclidean hierarchy in modal logic. Studia Logica, 75(3):327-344, 2003.

16. J. van Benthem, G. Bezhanishvili, B. ten Cate, and D. Sarenac. Multimodal logics of products of topologies. Studia Logica, 84(3):369-392, 2006.

17. J. van Benthem, N. Bezhanishvili, and I. Hodkinson. Sahlqvist correspondence for modal mu-calculus. Studia Logica, 100(1-2):31-60, 2012.

18. J. van Benthem, S. Ghosh, and F. Liu. Modelling simultaneous games in dynamic logic. Synthese, 165:247-268, 2008.

19. J. van Benthem, T. Hoshi, J. Gerbrandy, and E. Pacuit. Merging frameworks for interaction. Journal of Philosophical logic, 38(5):491-526, 2009.

20. J. van Benthem and F. Liu. Diversity of logical agents in games. Philosophia Scientice, 8(2):165-181, 2004.

21. J. van Benthem and E. Pacuit. The tree of knowledge in action: Towards a common perspective. In Advances in Modal Logic (AiML), 2006.

22. J. van Benthem and E. Pacuit. Dynamic logics of evidence-based beliefs. Studia Logica, pages 61-92, 2011.

23. J. van Benthem and D. Sarenac. The geometry of knowledge. In Aspects of universal logic, volume 17 of Travaux Log., pages 1-31. Univ. Neuchâtel, Neuchâtel, 2004.

24. J.van Benthem. Modal Logic and Classical Logic. Bibliopolis, Napoli, 1985.

25. G. Bezhanishvili, L. Esakia, and D. Gabelaia. Some results on modal axiomatization and definability for topological spaces. Studia Logica, 81(3):325-355, 2005.

26. G. Bezhanishvili, L. Esakia, and D. Gabelaia. The modal logic of Stone spaces: diamond as derivative. Rev. Symb. Log., 3(1):26-40, 2010.

27. G. Bezhanishvili, L. Esakia, and D. Gabelaia. Spectral and $T_{0}$-spaces in d-semantics. In Logic, Language, and Computation - 8th International Tbilisi Symposium on Logic, Language, and Computation. Revised Selected Papers, volume 6618 of LNAI, pages 16-29, 2011.

28. G. Bezhanishvili and M. Gehrke. Completeness of S4 with respect to the real line: revisited. Ann. Pure Appl. Logic, 131(1-3):287-301, 2005.

29. G. Bezhanishvili, R. Mines, and P. Morandi. Scattered, Hausdorff-reducible, and hereditarily irresolvable spaces. Topology Appl., 132(3):291-306, 2003.

30. G. Bezhanishvili, R. Mines, and P. Morandi. Topo-canonical completions of closure algebras and Heyting algebras. Algebra Universalis, 58(1):1-34, 2008.

31. N. Bezhanishvili. Pseudomonadic algebras as algebraic models of doxastic modal logic. MLQ Math. Log. Q., 48(4):624-636, 2002.

32. P. Blackburn, M. de Rijke, and Y. Venema. Modal Logic. Cambridge University Press, 2001.

33. P. Blackburn, M. de Rijke, and Y. Venema. Modal Logic, volume 53 of Cambridge Tracts in Theoretical Computer Science. Cambridge University Press, Cambridge, 2001.

34. J. Bradfield and C. Stirling. Modal mu-calculus. In P. Blackburn, J. van Benthem, and F. Wolter, editors, Handbook of Modal Logic, pages 721-756. Elsevier, 2007.

35. B. ten Cate, D. Gabelaia, and D. Sustretov. Modal languages for topology: expressivity and definability. Ann. Pure Appl. Logic, 159(1-2):146-170, 2009.

36. A. Chagrov and M. Zakharyaschev. Modal Logic. The Clarendon Press, 1997.

37. B. F. Chellas. Modal logic. Cambridge University Press, Cambridge, 1980.

38. A. Dabrowski, L. Moss, and R. Parikh. Topological reasoning and the logic of knowledge. Ann. Pure Appl. Logic, 78(1-3):73-110, 1996. 
39. H. van Ditmarsch, D. Duque Fernández, and W. van der Hoek. On the definability of simulability and bisimilarity by finite epistemic models. In J. Leite, P. Torroni, T. Ågotnes, G. Boella, and L. van der Torre, editors, Computational Logic in Multi-Agent Systems (CLIMA XII), volume 6814 of LNCS, pages 74-87, 2011.

40. H. van Ditmarsch, W. van der Hoek, and B. Kooi. Knowing more - from global to local correspondence. In C. Boutillier, editor, Proceedings of IJCAI-09, pages 955-960, 2009.

41. R. Engelking. General topology, volume 6. Heldermann Verlag, Berlin, second edition, 1989.

42. L. Esakia. Intuitionistic logic and modality via topology. Ann. Pure Appl. Logic, 127(13):155-170, 2004. Provinces of logic determined.

43. L. L. Esakia. Diagonal constructions, the Löb formula and rarefied Cantor's scattered spaces. In Studies in logic and semantics, pages 128-143. "Metsniereba", Tbilisi, 1981.

44. L. L. Esakia. Weak transitivity-a restitution. In Logical investigations, No. 8 (Russian) (Moscow, 2001), pages 244-255. "Nauka", Moscow, 2001.

45. M. Escardo. Domain theory in logical form. Electronic Notes in Theoretical Computer Science, 87:21-156, 2004.

46. R. Fagin, J. Y. Halpern, Y. Moses, and M. Y. Vardi. Reasoning about Knowledge. The MIT Press, Cambridge, Massachusetts, 1995.

47. D. Fernández-Duque. Absolute completeness of $\mathrm{S} 4_{u}$ for its measure-theoretic semantics. In Advances in modal logic. Volume 8, pages 100-119. Coll. Publ., London, 2010.

48. G. Fontaine. Modal fixpoint logic: some model theoretic questions. PhD thesis, University of Amsterdam, 2010.

49. T. French, W. van der Hoek, P. Iliev, and B.P. Kooi. Succinctness of epistemic languages. In T. Walsh, editor, IJCAI, pages 881-886, 2011.

50. D. Gabbay, A. Pnueli, S. Shelah, and J. Stavi. On the temporal analysis of fairness. In POPL'80, pages 163-173, 1980.

51. D. M. Gabbay, A. Kurucz, F. Wolter, and M. Zakharyaschev. Many-dimensional modal logics: theory and applications, volume 148 of Studies in Logic and the Foundations of Mathematics. North-Holland Publishing Co., Amsterdam, 2003.

52. D. Gabelaia. Modal definability in topology. Master's Thesis, Available as ILLC report: MoL-2001-10:, 2001.

53. D. Gabelaia, A. Kurucz, F. Wolter, and M. Zakharyaschev. Products of 'transitive' modal logics. J. Symbolic Logic, 70(3):993-1021, 2005.

54. K. Georgatos. Modal logics for topological spaces. ProQuest LLC, Ann Arbor, MI, 1993. Thesis (Ph.D.)-City University of New York.

55. K. Georgatos. Reasoning about knowledge on computation trees. In Logics in artificial intelligence (York, 1994), volume 838 of LNCS, pages 300-315. Springer, Berlin, 1994.

56. M. S. Gerson. An extension of $S 4$ complete for the neighbourhood semantics but incomplete for the relational semantics. Studia Logica, 34(4):333-342, 1975.

57. R. Goldblatt. An algebraic study of well-foundedness. Studia Logica, 44(4):423-437, 1985.

58. J.Y. Halpern and M.Y. Vardi. The complexity of reasoning about knowledge and time. i. lower bounds. Journal of Computer and System Sciences, 38(1):195 - 237, 1989.

59. H. H. Hansen. Monotonic modal logics. Master's Thesis, Available as: ILLC report: PP2003-24, 2003.

60. H. H. Hansen, C. Kupke, and E. Pacuit. Bisimulation for neighbourhood structures. In M. Haveraanen, U. Montanari, and T. Mossakoswki, editors, CALCO, number 4624 in LNCS, pages 279-293. Springer, 2007.

61. D. Harel, D. Kozen, and J. Tiuryn. Dynamic Logic. The MIT Press, Cambridge, Massachusetts, 2000. Foundations of Computing Series.

62. B. Heinemann. Topology and knowledge of multiple agents. In Advances in Artificial Intelligence, pages 1-10, 2008.

63. B. Heinemann. The Cantor space as a generic model of topologically presented knowledge. In $C S R$, pages 169-180, 2010.

64. B. Heinemann. Logics for multi-subset spaces. J. Appl. Non-Classical Logics, 20(3):219240, 2010. 
65. B. Heinemann. Using hybrid logic for coping with functions in subset spaces. Studia Logica, 94(1):23-45, 2010.

66. J. Hintikka. Knowledge and Belief, An Introduction to the Logic of the Two Notions. Cornell University Press, Ithaca, New York, 1962. republished in 2005 by King's College, London.

67. J. Hintikka. Reasoning about knowledge in philosophy. In J. Y. Halpern, editor, Proceedings of the 1986 Conference on Theoretical Aspects of Reasoning About Knowledge, pages 63-80, San Francisco, 1986. Morgan Kaufmann Publishers.

68. I. Hodkinson. Simple completeness proofs for some spatial logics of the real line. Proc. 12th Asian Logic Conference, Wellington, 2011. To appear.

69. I. Hodkinson. On the Priorean temporal logic with 'around now' over the real line. Submitted, 2012

70. W. van der Hoek. Systems for knowledge and beliefs. Journal of Logic and Computation, 3(2):173-195, 1993.

71. W. van der Hoek and J.-J. Ch Meyer. Making some issues of implicit knowledge explicit. International Journal of Foundations of Computer Science, 3(2):193-224, 1992.

72. K. D. Joshi. Introduction to general topology. A Halsted Press Book. John Wiley \& Sons Inc., New York, 1983.

73. R. Kontchakov, I. Pratt-Hartmann, F. Wolter, and Michael Zakharyaschev. Spatial logics with connectedness predicates. Log. Methods Comput. Sci., 6(3):3:5, 43, 2010.

74. S. Kraus and D. Lehmann. Knowledge, belief and time. Theoretical Computer Science, 58:155-174, 1988 .

75. P. Kremer. The incompleteness of $S 4 \oplus S 4$ for the product space $\mathbb{R} \times \mathbb{R}$. Studia Logica. To appear.

76. P. Kremer. Strong completeness of S4 wrt any dense-in-itself metric space. Review of Symbolic Logic. To appear.

77. P. Kremer. Strong completeness of S4 wrt the real line. Manuscript, 2012.

78. L. Lismont. La connaissance commune en logique modale. Mathematical Logic Quarterly, 39(1):115-130, 1993.

79. L. Lismont. Common knowledge: Relating anti-founded situation semantics to modal logic neighbourhood semantics. Journal of Logic, Language and Information, 3(4):285-302, 1994.

80. A. Lomuscio and M. Ryan. On the relation between interpreted systems and Kripke models. In Proceedings of the AI97 Workshop on Theoretical and Practical Foundations of Intelligent Agents and Agent-Oriented Systems, volume 1441 of LNAI, pages 46-59, 1997.

81. A. Lomuscio and M. Sergot. Investigations in grounded semantics for multi-agent systems specification via deontic logic, 2000. Technical Report, Imperial College, London.

82. J. Lucero-Bryan. Modal logics of some subspaces of the real numbers: Diamond as derivative. ProQuest LLC, Ann Arbor, MI, 2010. Thesis (Ph.D.)-New Mexico State University.

83. J. Lucero-Bryan. The d-logic of the rational numbers: a fruitful construction. Studia Logica, 97(2):265-295, 2011.

84. J. Lucero-Bryan. The d-logic of the real line. Journal of Logic and Computation, 23(1):121156, 2013.

85. J. C. C. McKinsey and A. Tarski. The algebra of topology. Ann. of Math. (2), 45:141-191, 1944.

86. J.-J. Ch. Meyer and W. van der Hoek. Epistemic Logic for AI and Computer Science, volume 41 of Cambridge Tracts in Theoretical Computer Science. Cambridge University Press, Cambridge, 1995.

87. G. Mints. A completeness proof for propositional S4 in Cantor space. In Logic at work, volume 24 of Stud. Fuzziness Soft Comput., pages 79-88. Physica, Heidelberg, 1999.

88. G. Mints and T. Zhang. A proof of topological completeness for $S 4$ in $(0,1)$. Ann. Pure Appl. Logic, 133(1-3):231-245, 2005.

89. R. C. Moore. Reasoning about knowledge and action. In Proceedings of the Fifth International Joint Conference on Artificial Intelligence (IJCAI-77), Cambridge, Massachusetts, 1977. 
90. L. Moss and R. Parikh. Topological reasoning and the logic of knowledge. In Proceedings of the 4th Conference on Theoretical Aspects of Reasoning about Knowledge, TARK, pages 95-105, 1992.

91. R. Parikh, L. Moss, and C. Steinsvold. Topology and epistemic logic. In Handbook of spatial logics, pages 299-341. Springer, Dordrecht, 2007.

92. R. Parikh and R. Ramanujam. Distributed processes and the logic of knowledge. In R. Parikh, editor, Logics of Programs, volume 193 of LNCS, pages 256-268. Springer Berlin / Heidelberg, 1985.

93. A. S. Rao and M. P. Georgeff. Modeling rational agents within a BDI-architecture. In R. Fikes and E. Sandewall, editors, Proceedings of Knowledge Representation and Reasoning (KR\&R-91), pages 473-484, San Francisco, April 1991. Morgan Kaufmann Publishers.

94. V. Shehtman. Derived sets in Euclidean spaces and modal logic. Report X-1990-05, University of Amsterdam, 1990.

95. V. Shehtman. A logic with progressive tenses. In M. de Rijke, editor, Diamonds and defaults, pages 255-285. Kluwer Academic Publishers, 1993.

96. V. Shehtman. "Everywhere" and "here". J. Appl. Non-Classical Logics, 9(2-3):369-379, 1999. Issue in memory of George Gargov.

97. M. B. Smyth. Topology. In Handbook of logic in computer science, Vol. 1, volume 1 of Handb. Log. Comput. Sci., pages 641-761. Oxford Univ. Press, New York, 1992.

98. C. Steinsvold. Topological models of belief logics. ProQuest LLC, Ann Arbor, MI, 2007. Thesis (Ph.D.)-City University of New York.

99. K. Su, A. Sattar, G. Governatori, and Q. Chen. A computationally grounded logic of knowledge, belief and certainty. In In Proceedings of AAMAS, pages 149-156. ACM Press, 2005.

100. A. S. Troelstra and D. van Dalen. Constructivism in mathematics. Vols. I and II. Studies in Logic and the Foundations of Mathematics. North-Holland Publishing Co., Amsterdam, 1988. An introduction.

101. S. Vickers. Topology via logic, volume 5 of Cambridge Tracts in Theoretical Computer Science. Cambridge University Press, Cambridge, 1989.

102. Y. Wang and T. Ågotnes. Multi-agent subset space logic. Accepted for IJCAI 2013, 2013.

103. F. Wolter and M. Zakharyaschev. Spatial reasoning in RCC-8 with Boolean region terms. In Proceedings of the 14th European Conference on Artificial Intelligence (ECAI-2000), pages 244-248, Berlin, 2000. IOS Press.

104. M. Wooldridge. Computationally grounded theories of agency. In E. Durfee, editor, Proc. ICMAS, pages 13-20. IEEE Press, 2000. 